\title{
Automaton Semigroups and Groups: On the Undecidability of Problems Related to Freeness and Finiteness
}

\author{
Daniele D’Angeli*1 ${ }^{*}$ Emanuele Rodaro ${ }^{\dagger 2}$ and Jan Philipp Wächter ${ }^{3}$ \\ ${ }^{1}$ Institut für Diskrete Mathematik, Technische Universität Graz, Steyrergasse 30, $8010 \mathrm{Graz}$, Austria
${ }^{2}$ Department of Mathematics, Politecnico di Milano, Piazza Leonardo da Vinci, 32, 20133 Milano, Italy \\ ${ }^{3}$ Institut für Formale Methoden der Informatik (FMI), Universität Stuttgart, Universitätsstraße 38, 70569 Stuttgart, Germany
}

April 10, 2020

\begin{abstract}
In this paper, we study algorithmic problems for automaton semigroups and automaton groups related to freeness and finiteness. In the course of this study, we also exhibit some connections between the algebraic structure of automaton (semi)groups and their dynamics on the boundary.

First, we show that it is undecidable to check whether the group generated by a given invertible automaton has a positive relation, i. e. a relation $\boldsymbol{p}=\mathbb{1}$ such that $\boldsymbol{p}$ only contains positive generators. Besides its obvious relation to the freeness of the group, the absence of positive relations has previously been studied by the first two authors and is connected to the triviality of some stabilizers of the boundary. We show that the emptiness of the set of positive relations is equivalent to the dynamical property that all (directed positive) orbital graphs centered at non-singular points are acyclic. Our approach also works to show undecidability of the freeness problem for automaton semigroups, which negatively solves an open problem by Grigorchuk, Nekrashevych and Sushchansky. In fact, we show undecidability of a strengthened version where the input automaton is complete and invertible.

Gillibert showed that the finiteness problem for automaton semigroups is undecidable. In the second part of the paper, we show that this undecidability result also holds if the input is restricted to be bi-reversible and invertible (but, in general, not complete). As an immediate consequence, we obtain that the finiteness problem for automaton subsemigroups of semigroups generated by invertible, yet partial automata, so called automaton-inverse semigroups, is also undecidable.
\end{abstract}

\footnotetext{
${ }^{*}$ The first author was supported by the Austrian Science Fund projects FWF P24028-N18 and FWF P29355-N35.

${ }^{\dagger}$ The second author thanks the project INDAM-GNSAGA.
} 


\section{Introduction}

Automaton groups, i.e. groups generated by synchronous Mealy automata, are a quite intriguing class of groups. They have deep connections with many areas of mathematics, from the theory of profinite groups to complex dynamics and theoretical computer science, and they serve as a source of examples or counterexamples for many important group theoretic problems (see e.g. [40] for an introduction). Despite these connections and the many surprising and interesting consequences, knowledge about the class of automaton groups from the algebraic, algorithmic and dynamical perspective is still rather limited. From the algorithmic point of view, the word problem for automaton groups is decidabl $\left.\right|^{1}$ while many other problems are suspected (and sometimes proven) to be undecidable. In this regard, the most studied problems in the literature are the finiteness problem, the freeness problem and the conjugacy problem. The latter has been proven to be undecidable by Šunić and Ventura [38]. However, if this problem is restricted to the contracting case, then it turns out to be decidable, see [5]. Decidability of the finiteness problem for automaton groups is still open. However, some partial results are known when certain properties of the generating automaton are relaxed: Belk and Bleak showed undecidability of the finiteness problem for groups generated by asynchronous automata [3] and Gillibert showed undecidability of the finiteness problem for automaton semigroups [20, which are generated by synchronous but not necessarily invertible Mealy automata. Other results worth mentioning in this respect are the recent proof by Gillibert showing undecidability of the order problem of automaton groups [21] and the result by Bartholdi and Mitrofanov that the order problem is already undecidable for contracting automaton groups [2]. On the other side, Klimann showed that the finiteness problem is solvable for reversible, invertible automata with two states or two letters and that so is the freeness problem for semigroups generated by two state invertible, reversible automata [28]. Obviously, it is usually easier to show undecidability results for automaton semigroups than it is for automaton groups. This might be one of the reasons why the less studied class of automaton semigroups seems to stir up more interest lately. There is, for example, the semigroup theoretic work of Cain [9], and Brough and Cain [6, 7], approaches to semigroups via duals of automata generating groups [14, 15] or studies of the torsion-freeness of automaton semigroups (see e.g. [22]; also for some further references). Other works study the existence of free subsemigroups in automaton groups and semigroups (see e. g. [13, 14, 15, 19, 33, 37]).

The aim of this paper is to make a contribution to the algorithmic point of view, in particular to the finiteness problem and the freeness problem for automaton (semi)groups. We approach these problems by making use of ideas with a dynamical flavor [23] underlining the importance of the dynamics on the boundary when considering algorithms: as an alternative to the automaton definition, one can also view automaton (semi)groups as level-preserving (finite-state) actions on the rooted infinite tree $\Sigma^{*}$ corresponding to the finite words over some alphabet $\Sigma$; the limit of this tree (in the sense of GromovHausdorff convergence) is $\Sigma^{\omega}$, which corresponds to the right-infinite words over $\Sigma$ and

\footnotetext{
${ }^{1}$ See [16] for some discussion of its complexity.
} 
on which the group also acts. As automaton (semi)groups are finitely generated and, thus, countable, their action on $\Sigma^{\omega}$ cannot be transitive. In this context, one of the main problems in the combinatorial study of automaton groups is to understand the (boundary) orbital Schreier graphs of a given automaton group [10]. It is natural to ask which properties of a group can be recognized by exploring the structure of these Schreier graphs. Of course, this idea can be extended to semigroups by considering their orbital graphs on the boundary.

After introducing some preliminaries, we first consider the freeness problem for automaton (semi)groups. Inspired by links between the freeness of an automaton group and the dynamics on periodic points in the boundary of the dual automaton exhibited in [14, 15], we discuss the problem of checking whether a given automaton generating a group yields a positive relation, i. e. a relation $\boldsymbol{q}=\mathbb{1}$ where $\boldsymbol{q}$ contains only positive generators, which was previously studied in [12]. In Proposition 3.4, we first show that the existence of a positive relation is equivalent to having a cycle in some boundary Schreier graph. Then, we proceed to show undecidability of the existence of a positive relation; a problem left open in [12, Problem 3]. We do this by reducing ICP, an undecidable [4, Theorem 11] problem similar to Post's Correspondence Problem, to the problem using a construction given in [38. We observe that, using the same approach, one can reduce IICP, a variation of ICP of unknown decidability, to the freeness problem of automaton groups. Furthermore, we show using a similar reduction that (even a strengthened version of) the freeness problem for automaton semigroups is undecidable in Theorem 3.12 and Corollary 3.13 .

In the second part of the paper, we deal with the finiteness problem for automaton groups. In the spirit of [20] and [12], we attack the problem using Wang tilings. First, we show that finiteness of an automaton (semi)group is characterized by uniform boundedness of the orbital graphs in the boundary in Proposition 4.1. Afterwards, in Proposition 4.6, we connect the finiteness of a semigroup generated by a (partial) automaton obtained from a Wang tile set with the existence of non-periodic tiling, which is the crucial point of reducing the tiling problem for 4-way deterministic tiles (proved undecidable by Lukkarila [30]) to a strengthened version of the finiteness problem for automaton semigroups where the input automaton is known to be bi-reversible and invertible, which is stated to be undecidable in Theorem 4.7. The strengthened version is already quite close to the group case; the only missing part is completeness of the automaton. An immediate consequence is that it is undecidable to check whether a subsemigroup of an automaton-inverse semigroup (i. e. the inverse semigroup generated by an invertible, yet partial automaton) is finite.

\section{Preliminaries}

Fundamentals. We use $A \sqcup B$ to denote the disjoint union of two sets $A$ and $B$. The set $\mathbb{N}$ is the set of natural numbers including $0, \mathbb{Z}$ is the set of integers and $\mathbb{Z}_{+}$is the set of (strictly) positive integers. Finally, $\mathbb{R}$ is the set of real numbers.

An alphabet $\Sigma$ is a non-empty finite set of letters. By $\Sigma^{*}$, we denote the set of all 
finite words over the alphabet $\Sigma$, including the empty word, which we denote by $\varepsilon$. Furthermore, we set $\Sigma^{+}=\Sigma^{*} \backslash\{\varepsilon\}$ and use the notation $\Sigma^{n}$ for the set of words of length $n$ over $\Sigma$ and the notation $\Sigma^{\leq n}$ for the set of words $w$ of length up to $|w|=n$; $\Sigma^{<n}$ is the set of words of length smaller than $n$, respectively. The set of $\omega$-words, i. e. infinite words whose positions are the natural numbers, is denoted by $\Sigma^{\omega}$. Whenever we refer to a word in this paper, it can either be a finite word or an $\omega$-word. The set $\Sigma^{*} \cup \Sigma^{\omega}$ is a metric space and, thus, also a topological space. The distance between two distinct words $x$ and $y$ is $|\Sigma|^{-|p|}$ where $p$ is the longest common prefix $\left.\right|^{2}$ of $x$ and $y$. Notice that this metric induces the discrete topology on $\Sigma^{*}, \Sigma^{n}$ and $\Sigma \leq n$ for all $n \in \mathbb{N}$. On $\Sigma^{\omega}=\prod_{i \in \mathbb{N}} \Sigma^{1}$, it induces Tychonoff's topology. The sequence $\left(\Sigma^{\leq n}\right)_{n \in \mathbb{N}}$ of compact metric spaces converges to $\Sigma^{*} \cup \Sigma^{\omega}$ (in the sense of Gromov-Hausdorf ${ }^{3}$ ); therefore, $\Sigma^{\omega}$ is often referred to as the boundary of $\Sigma^{*}$.

On the algebraic side, we will be dealing with semigroups (and monoids), inverse semigroups and groups. In this context, we want to remind the reader of the difference between inverses in semigroups and in groups. An element $\bar{s}$ of a semigroup $S$ is called (semigroup) inverse to another element $s \in S$ if $\bar{s} s \bar{s}=\bar{s}$ and $s \bar{s} s=s$ hold. On the other hand, we will call an element $m^{-1}$ of a monoid $M$ (which may be a group) the group inverse of $m \in M$ if we have $m^{-1} m=m m^{-1}=\mathbb{1}$. Here and throughout this paper, we use $\mathbb{1}$ to denote the neutral element of a monoid (or group, of course); the monoid in question will be clear from the context. Clearly, the group inverse of a monoid element is always also a (semigroup) inverse. The converse does not hold in general, however. To emphasize this difference, we use the notation $\bar{m}$ to denote (semigroup) inverses and the notation $m^{-1}$ to denote group inverses.

A semigroup $S$ is an inverse semigroup if every element $s \in S$ has a unique inverse $\bar{s}$, see [25, 34] for further details on inverse semigroups. As the Preston-Vagner Theorem (see [25, p. 150] or [34, p. 168]) demonstrates, there is a close connection between inverse semigroups and partial function (or partial maps). For these, we fix some notation. To indicate that a function $f$ from a set $A$ to a set $B$ is partial, we write $f: A \rightarrow_{p} B$. The domain of $f$, denoted by $\operatorname{dom} f$, is the subset of $A$ on which $f$ is defined. If we have $\operatorname{dom} f=A$, i. e. that $f$ is defined on all elements in $A$, then we call $f$ a total function from $A$ to $B$ and write $f: A \rightarrow B$. The counter part to $\operatorname{dom} f$ is $\operatorname{im} f$, the image of $f$; it consists of the images under $f$ of the elements in $\operatorname{dom} f$. We say that a partial functions $\bar{f}: B \rightarrow_{p} A$ is inverse to another partial function $f: A \rightarrow_{p} B$ if $\operatorname{dom} \bar{f}=\operatorname{im} f$, $\operatorname{im} \bar{f}=\operatorname{dom} f$ and $f(\bar{f}(f(a)))=f(a)$ for all $a \in \operatorname{dom} f$ as well as $\bar{f}(f(\bar{f}(b)))=\bar{f}(b)$ for all $b \in \operatorname{im} f$; in an abuse of terminology, we say that $f^{-1}=\bar{f}$ is a group inverse of $f$ if $\operatorname{dom} f^{-1}=\operatorname{im} f, \operatorname{im} f^{-1}=\operatorname{dom} f$ and $f^{-1}(f(a))=a$ for all $a \in \operatorname{dom} f$ as well as $f\left(f^{-1}(b)\right)=b$ for all $b \in \operatorname{im} f$. Note that in the latter case both functions are injective and the group inverse is unique.

Automata: Definition, Properties and Operations. In this paper, the term automaton refers to a special form of a finite-state transducer: it is

\footnotetext{
${ }^{2} \mathrm{~A}$ word $w$ is a prefix of a word $x$ if there is a word $y$ with $w y=x$.

${ }^{3}$ For Gromov-Hausdorff convergence, see [24].
} 
- synchronous (it outputs exactly one letter on input of one letter),

- not necessarily complete and

- its input and output alphabets coincide.

Formally, let $Q$ be a non-empty set and let $\Sigma$ be an alphabet. An automaton is a triple $\mathcal{T}=(Q, \Sigma, \delta)$ with $\delta \subseteq Q \times \Sigma \times \Sigma \times Q$. The set $Q$ is called the state set of $\mathcal{T}, \Sigma$ is its (input and output) alphabet and $\delta$ is its transition set. Accordingly, an element $(q, a, b, p) \in \delta$ is called a transition of the automaton from state $q$ on input $a$ with output $b$ into state $p$. To denote a transition, we use the more graphical notation $q \stackrel{a / b}{\longrightarrow} p$ instead of the tuple notation $(q, a, b, p)$. Additionally, we use the common graphical representation for automata: a transition $q \stackrel{a / b}{\longrightarrow} p \in \delta$ is represented as

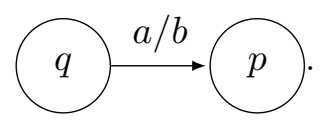

An automaton $\mathcal{T}=(Q, \Sigma, \delta)$ is called deterministic if

$$
|\{q \stackrel{a / b}{\longrightarrow} p \mid q \stackrel{a / b}{\longrightarrow} p \in \delta, b \in \Sigma, p \in Q\}| \leq 1
$$

holds for all $q \in Q$ and all $a \in \Sigma$. It is called complete if we have

$$
|\{q \stackrel{a / b}{\longrightarrow} p \mid q \stackrel{a / b}{\longrightarrow} p \in \delta, b \in \Sigma, p \in Q\}| \geq 1
$$

for all $q \in Q$ and all $a \in \Sigma$. An automaton $\mathcal{T}=(Q, \Sigma, \delta)$ is reversible if

$$
|\{q \stackrel{a / b}{\longrightarrow} p \mid q \stackrel{a / b}{\longrightarrow} p \in \delta, b \in \Sigma, q \in Q\}| \leq 1
$$

holds for all $a \in \Sigma$ and $p \in Q$ (i.e. if it is co-deterministic with respect to the input). This means that $q \stackrel{a / b}{\longrightarrow} p, q^{\prime} \stackrel{a / b^{\prime}}{\longrightarrow} p \in \delta$ implies $q=q^{\prime}$ and $b=b^{\prime}$ for every $a, b, b^{\prime} \in \Sigma$ and every $q, q^{\prime}, p \in Q$.

For every automaton $\mathcal{T}=(Q, \Sigma, \delta)$, one can define its inverse automaton $\overline{\mathcal{T}}=(\bar{Q}, \Sigma, \bar{\delta})$ where $\bar{Q}$ is a disjoint copy of $Q$ and we have

$$
\bar{q} \stackrel{b / a}{\longrightarrow} \bar{p} \in \bar{\delta} \Longleftrightarrow q \stackrel{a / b}{\longrightarrow} p \in \delta,
$$

i. e. we swap input and output. By defining $\overline{\bar{q}}=q$ for all $q \in Q$, we obtain that taking the inverse of an automaton is an involution, i. e. we have $\overline{\overline{\mathcal{T}}}=\mathcal{T}$. For any of the automaton properties defined above, we also have an inverse version: we say $\mathcal{T}$ is inversedeterministic (sometimes also called invertible) if $\overline{\mathcal{T}}$ is deterministic, it is inverse-complete if $\overline{\mathcal{T}}$ is complete, and it is inverse-reversible if $\overline{\mathcal{T}}$ is reversible (this is the case if $\mathcal{T}$ is co-deterministic with respect to the output). Additionally, we define versions of the properties which describe that they hold for an automaton and its inverse at the same time: an automaton $\mathcal{T}$ is bi-deterministic if it is deterministic and inverse-deterministic, it is bi-complete if it is complete and inverse-complete and it is bi-reversible if it is reversible and inverse-reversible. 
Next to the inverse of an automaton, there is also its dual. For an automaton $\mathcal{T}=$ $(Q, \Sigma, \delta)$, we define its dual $\partial \mathcal{T}=(\Sigma, Q, \partial \delta)$ by

$$
a \stackrel{q / p}{\longrightarrow} b \in \partial \delta \Longleftrightarrow q \stackrel{a / b}{\longrightarrow} p \in \delta,
$$

i.e. we swap the roles of states and letters. Just like taking the inverse, taking the dual is an involution: $\partial \partial \mathcal{T}=\mathcal{T}$. Notice that there are many connections between $\mathcal{T}$ and its dual. For example, we have that

- $\mathcal{T}$ is deterministic if and only if $\partial \mathcal{T}$ is deterministic,

- $\mathcal{T}$ is complete if and only if $\partial \mathcal{T}$ is complete,

- $\mathcal{T}$ is inverse-deterministic if and only if $\partial \mathcal{T}$ is reversible, and that

- $\mathcal{T}$ is inverse-reversible if and only if $\partial \mathcal{T}$ is inverse-reversible.

Other operations on automata involve two (or more) automata. For example, for any two automata $\mathcal{T}_{1}=(Q, \Sigma, \delta)$ and $\mathcal{T}_{2}=(P, \Gamma, \rho)$, one can take their union automaton $\mathcal{T}_{1} \cup \mathcal{T}_{2}=(Q \cup P, \Sigma \cup \Gamma, \delta \cup \rho)$. For the union of an automaton $\mathcal{T}=(Q, \Sigma, \delta)$ and its inverse $\overline{\mathcal{T}}=(\bar{Q}, \Sigma, \bar{\delta})$, we use a shorthand notation and denote it by $\widetilde{\mathcal{T}}=(\widetilde{Q}, \Sigma, \widetilde{\delta})$, where $\widetilde{Q}=Q \cup \bar{Q}$ and $\widetilde{\delta}=\delta \cup \bar{\delta}$.

In addition to the union, one can also take the composition of two automata $\mathcal{T}_{1}=$ $(Q, \Sigma, \delta)$ and $\mathcal{T}_{2}=(P, \Sigma, \rho)$. It is the automaton $\mathcal{T}_{2} \circ \mathcal{T}_{1}=(P \circ Q, \Sigma, \rho \circ \delta)$ with $P \circ Q=$ $\{p \circ q \mid p \in P, q \in Q\}$ (a formal copy of $P \times Q$ ) given by

$$
p \circ q \stackrel{a / c}{\longrightarrow} p^{\prime} \circ q^{\prime} \in \rho \circ \delta \Longleftrightarrow q \stackrel{a / b}{\longrightarrow} q^{\prime} \in \delta, p \stackrel{b / c}{\longrightarrow} p^{\prime} \in \rho \text { for some } b \in \Sigma .
$$

The idea is to use the output $b$ of $\mathcal{T}_{1}$ on input of $a$ as input for the second automaton $\mathcal{T}_{2}$. Notice that composition preserves the properties defined above: if $\mathcal{T}_{1}$ and $\mathcal{T}_{2}$ are deterministic/complete/reversible, then so is $\mathcal{T}_{1} \circ \mathcal{T}_{2}$.

A special form of the composition of automata, is the $k^{\text {th }}$-power $\mathcal{T}^{k}$ of an automaton $\mathcal{T}=(Q, \Sigma, \delta)$ for some $k \geq 1$. It is the $k$-fold composition of $\mathcal{T}$ with itself:

$$
\mathcal{T}^{k}=\underbrace{\mathcal{T} \circ \mathcal{T} \circ \cdots \circ \mathcal{T}}_{k \text { times }} \text {. }
$$

Semantics of Automata, Automaton Semigroups. So far, we have defined automata only formally. Of course, behind these definitions stands the common intuitive understanding that an automaton emits an output for some input. Since we will be dealing primarily with deterministic automata in this paper, we give this class a special name: a deterministic automaton is called an $S$-automaton from now on. This name stems from the fact that these automata generate semigroups (as we will see shortly). Later on, we will also encounter $\bar{S}$-automata (generating inverse semigroups) and $G$-automata (generating groups). Every $S$-automaton $\mathcal{T}=(Q, \Sigma, \delta)$ induces a partial right action $\cdot: \Sigma \times Q \rightarrow_{p} Q$ of $\Sigma$ on $Q$ : we have $q \cdot a=p$ if $q \stackrel{a / b}{\longrightarrow} p \in \delta$ for some $b \in \Sigma$; here, we use the more common infix notation for $\cdot$. Notice that $b$ must be unique since $\mathcal{T}$ is 


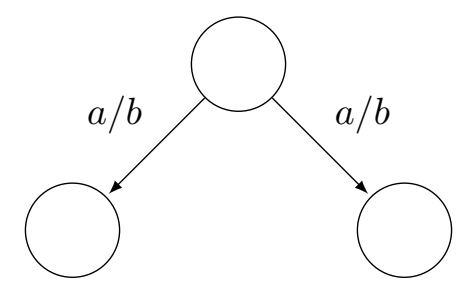

Figure 1: A bi-reversible automaton which is neither deterministic nor inversedeterministic

deterministic by definition. This action can be extended into a partial action of $\Sigma^{*}$ on $Q$ by setting $q \cdot \varepsilon=q$ and $q \cdot a_{1} a_{2} \ldots a_{n}=\left(\left(q \cdot a_{1}\right) \cdot a_{2}\right) \ldots \cdot a_{n}$ (if defined). Intuitively, this action describes reading the finite input word in the automaton: if one starts reading the finite word $w$ in state $q$, one ends up in $q \cdot w$. Notice that this action is total, i. e. - is a function $\Sigma^{*} \times Q \rightarrow Q$, if (and only if) $\mathcal{T}$ is complete.

Additionally, every $S$-automaton $\mathcal{T}$ induces a partial left action $\circ: Q \times \Sigma \rightarrow_{p} \Sigma$ of $Q$ on $\Sigma$ : we have $q \circ a=b$ if $q \stackrel{a / b}{\longrightarrow} p \in \delta$ for some $p \in Q$; again, we use infix notation for $\circ$. This action can be extended into an action of $Q$ on $\Sigma^{*}$ by setting $q \circ \varepsilon=\varepsilon$ and $q \circ a w=(q \circ a)[(q \cdot a) \circ w]$ (if defined) for all $a \in \Sigma$ and $w \in \Sigma^{+}$. Here, the intuition is to read a finite input word $a_{1} \ldots a_{n}$ in the automaton starting in state $q$. After each letter, the automaton emits an output letter and transitions into a new state. From this state onwards, the next letter is read and so on. Notice that this action is also total, i. e. $\circ$ is a function $Q \times \Sigma^{*} \rightarrow \Sigma^{*}$, if (and only if) $\mathcal{T}$ is complete. Furthermore, notice that $\circ$ can be extended into a (partial) function $Q \times \Sigma^{\omega} \rightarrow_{p} \Sigma^{\omega}$.

We can also consider the action of each individual state $q$. We re-use the notation $q \circ$ to denote the function $q \circ: \Sigma^{*} \cup \Sigma^{\omega} \rightarrow_{p} \Sigma^{*} \cup \Sigma^{\omega}$ induced by $\circ$ with first parameter $q$. Notice that, because the automaton is synchronous, all functions $q \circ$ are length-preserving (whenever they are defined) and prefix-compatible. Now, we can consider the closure of the functions $Q \circ=\{q \circ \mid q \in Q\}$ for some $S$-automaton $\mathcal{T}=(Q, \Sigma, \delta)$ under (finite) composition of partial functions. This is the semigroup generated by $\mathcal{T}$, which we denote by $\mathscr{S}(\mathcal{T})$. A semigroup is called an automaton semigroup if it is generated by some $S$-automaton $\mathcal{T}$. To avoid notational overhead, we omit the o symbols in an element $\boldsymbol{q} \circ=q_{n} \circ \cdots \circ q_{1} \circ$ of an automaton semigroup and simply write $\boldsymbol{q} \circ=q_{n} \ldots q_{1} \circ$.

Just like we extended the notation $q \circ u$ to cover more than a single state, we can do the same with the notation $q \cdot u$ : for an $S$-automaton $\mathcal{T}=(Q, \Sigma, \delta)$, a finite word $u \in \Sigma^{*}$ and states $q_{n}, \ldots, q_{2}, q_{1} \in Q$, define $q_{n} \ldots q_{2} q_{1} \cdot u=\left[q_{n} \ldots q_{2} \cdot\left(q_{1} \circ u\right)\right]\left(q_{1} \cdot u\right)$ inductively; furthermore, we define $\varepsilon \cdot u=\varepsilon$. Notice that $q_{n} \ldots q_{2} q_{1} \cdot u$ with this definition coincides with the state reached in $\mathcal{T}^{n}$ if one starts reading the finite input word $u$ in state $q_{n} \circ \cdots \circ q_{2} \circ q_{1}$.

Remark 2.1. Notice that the definition of an automaton semigroup presented here differs from the more common one, which is based on complete $S$-automata $4^{4}$. We call an automaton semigroup which is generated by a complete $S$-automaton a complete automaton

\footnotetext{
${ }^{4}$ This extended definition was previously used in [16].
} 
semigroup to distinguish the two concepts. There are obviously some connections between the two classes but it is not clear whether they coincide or not (see [17, Section 3] for a discussion of this). The usual way of going from automaton semigroups to complete automaton semigroups is to algebraically adjoin a zero element. For a semigroup $S$, let $S^{0}$ denote the semigroup obtained from $S$ by adjoining a new zero element. With this notation, if $S$ is an automaton semigroup, then $S^{0}$ is a complete automaton semigroup [16. Proposition 1] (but also note the discussion in [17, Section 3]).

Notice that non-complete automata sometimes behave differently compared to complete automata. For example, for them, reversibility does not imply determinism as can be seen in Figure 1.

Inverse Automaton Semigroups and Automaton Groups. Using non-complete automata to define automaton semigroups allows us to define automaton-inverse semigroups, an intermediate step between automaton semigroups and automaton groups 5 To do so, we introduce the name $\bar{S}$-automaton to denote bi-deterministic automata. For an $\bar{S}$-automaton $\mathcal{T}=(Q, \Sigma, \delta)$ and its inverse $\overline{\mathcal{T}}=(\bar{Q}, \Sigma, \bar{\delta})$, we can consider the set $\widetilde{Q} \circ=(Q \circ) \cup(\bar{Q} \circ)=\{\widetilde{q} \circ \mid \widetilde{q} \in Q \cup \bar{Q}=\widetilde{Q}\}$ of actions induced by the states of the automaton and its inverse. The inverse semigroup generated by $\mathcal{T}$, denoted by $\overline{\mathscr{S}}(\mathcal{T})$, is the closure of $\widetilde{Q} \circ$ under finite composition of partial functions. Notice that it is equal to the semigroup generated by the disjoint union of $\mathcal{T}$ and its inverse $\overline{\mathcal{T}}$, i. e. we have $\overline{\mathscr{S}}(\mathcal{T})=\mathscr{S}(\mathcal{T} \sqcup \overline{\mathcal{T}})$. A semigroup is called an automaton-inverse semigroup if it is generated by some $\bar{S}$-automaton. The name comes from the fact that $\bar{q} \circ$ and $q \circ$ are mutually inverse in the sense of partial functions for all states $q \in Q: q \circ \bar{q} \circ q \circ=q \circ$ and $\bar{q} \circ q \circ \bar{q} \circ=\bar{q} \circ$. Notice that, therefore, $\overline{\mathscr{S}}(\mathcal{T})$ is an inverse semigroup for all $\bar{S}$ automata $\mathcal{T}$. Please note that the use of $\bar{s}$ to denote the inverse of $s$ in a semigroup is compatible with the notation $\bar{q}$ for the corresponding state in the inverse automaton. Also note the difference in definition between an automaton-inverse semigroup and an inverse automaton semigroup 6

Example 2.2. The (finite) Brandt semigroup $B_{2}$ is generated by the elements $p$ and $q$ with the relations $p^{2}=q^{2}=0, p q p=p$ and $q p q=q$; it, thus, contains the elements $\{p, q, p q, q p, 0\}[25$, p. 32]7. To realize this semigroup as an automaton semigroup, we can let it act on itself (see the proof of [9, Proposition 4.6]). This leads to the $S$-automaton $\mathcal{T}=(\{q, p\},\{a, b, a b, b a, 0\}, \delta)$ depicted in Figure 2. Here, we have used the alphabet $\{a, b, a b, b a, 0\}$ (where we consider $a b$ and $b a$ to be single letters) instead of the elements of the semigroup to have a clearer distinction between the two concepts; the idea is to interpret $p$ as $a$ and $q$ as $b$. While the Brandt semigroup $\mathscr{S}(\mathcal{T})$ is an inverse semigroup with $\bar{q}=p$ and $\bar{p}=q$, the automaton $\mathcal{T}$ is not an $\bar{S}$-automaton as it is not inversedeterministic. Therefore, $\overline{\mathscr{S}}(\mathcal{T})$ has no defined meaning.

\footnotetext{
${ }^{5}$ It seems that the concept of inverse semigroups generated by partial automata has not been studied widely yet. However, it does appear in [31, for example, and a similar concept was studied by Olijnyk, Sushchansky and Slupik [32] (see also there for previous work by Sushchansky and Slupik).

${ }^{6}$ However, it turns out that both concepts coincide [17, Theorem 25].

${ }^{7}$ Readers familiar with syntactic semigroups might also find it interesting that $B_{2}$ is the syntactic semigroup of $\left\{(p q)^{n} \mid n \geq 1\right\}$.
} 


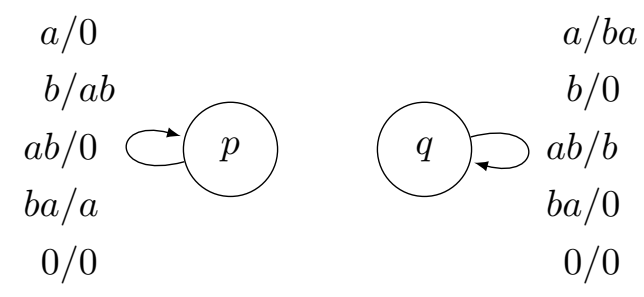

Figure 2: An automaton generating the Brandt semigroup $B_{2}$.

Finally, we call a complete $\bar{S}$-automaton a $G$-automaton. Notice that, by reasons of cardinality, any $G$-automaton is not only complete but also bi-complete. Thus, for a $G$-automaton $\mathcal{T}=(Q, \Sigma, \delta)$ with inverse $\overline{\mathcal{T}}=(Q, \Sigma, \delta)$, all functions $q \circ$ and $\bar{q} \circ$ with $q \in Q$ are total length-preserving bijections $\Sigma^{*} \rightarrow \Sigma^{*}$ (or $\Sigma^{\omega} \rightarrow \Sigma^{\omega}$, respectively). In this case, the automaton-inverse semigroup $\overline{\mathscr{S}}(\mathcal{T})$ generated by $\mathcal{T}$ (seen as an $\bar{S}$-automaton) is a group. We call it the group generated by $\mathcal{T}$ and denote it by $\mathscr{G}(\mathcal{T})$ to emphasize this fact. We call a group an automaton group if it is generated by some $G$-automaton $\mathcal{T}$.

We also use the (less-precise) name of an automaton structure to denote an automaton semigroup, an automaton-inverse semigroup or an automaton group. We summarize the definitions from above in Table 1 .

\begin{tabular}{lll} 
Automaton Class & Properties & Generated Structure \\
\hline$S$-automaton & deterministic & semigroup $\mathscr{S}(\mathcal{T})$ \\
$\bar{S}$-automaton & bi-deterministic & inverse semigroup $\mathscr{\mathscr { S }}(\mathcal{T})$ \\
$G$-automaton & bi-deterministic, bi-complete & group $\mathscr{G}(\mathcal{T})$
\end{tabular}

Table 1: Structures defined by automata

Example 2.3. The automaton

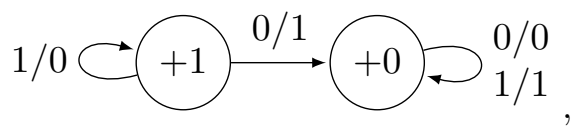

which shall be denoted by $\mathcal{T}$ for this example, is called the adding machine. It is a $G$ automaton and, as such, it is also an $\bar{S}$ - and an $S$-automaton. To understand the group and the semigroup generated by $\mathcal{T}$, it is useful to study the actions of +1 and +0 .

The state +0 obviously acts as the identity on $\{0,1\}^{*} \cup\{0,1\}^{\omega}$. The action $+1 \circ$ is more interesting. We can see any finite word from $\{0,1\}^{*}$ as representing a binary number in reverse (i.e. with least significant bit first). The same is true for $\omega$-words of the form $w 0^{\omega}$ for some $w \in\{0,1\}^{*}$ as the infinitely many 0 s at the end can be considered leading 0 s in the binary representation. Now, the action of +1 increments the encoded number by one. For example, we have $+1 \circ 010=110$ and $+1 \circ 1100^{\omega}=0010^{\omega}$. Therefore, it is 
not surprising that $\mathscr{S}(\mathcal{T})$, the semigroup generated by $\mathcal{T}$, is the free monoid with one generator. Similarly, $\mathscr{G}(\mathcal{T})$ is the free group with one generator.

If we extend 8 the adding machine into the automaton

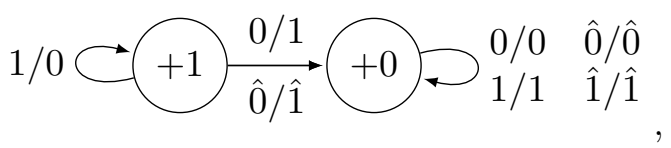

which we denote by $\hat{\mathcal{T}}$, then the result is not complete anymore but it still is an $\bar{S}$ automaton whose inverse is

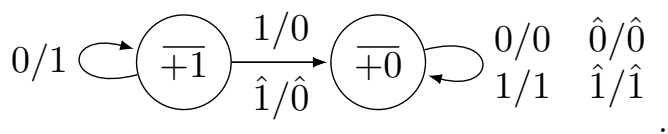

Notice that we have $\overline{+0} \circ=+0 \circ$. Similarly to our previous examples, $\overline{\mathscr{S}}(\hat{\mathcal{T}})$, the inverse semigroup generated by $\hat{\mathcal{T}}$, is the free inverse monoid with one generator. By [34, VIII.4.6, p. 381] (see also [32, Lemma 24]), this follows if we show $+1 \overline{+1}(\overline{+1})^{n}(+1)^{n} \neq(\overline{+1})^{n}(+1)^{n}$ and $\overline{+1}+1(+1)^{n}(\overline{+1})^{n} \neq(+1)^{n}(\overline{+1})^{n}$ for all $n \in \mathbb{N}$. We have $(+1)^{n} \circ 0^{n} \hat{0}=w \hat{0}$ where $w$ is the (reverse/least significant bit first) binary representation of the number $n$ with length $n$. Thus, we also have $(\overline{+1})^{n} \circ w \hat{0}=0^{n} \hat{0}$. On the other hand, $\overline{+1} \circ 0^{n} \hat{0}$ is undefined. This shows $+1 \overline{+1}(\overline{+1})^{n}(+1)^{n} \circ \neq(\overline{+1})^{n}(+1)^{n} \circ$. To show the other inequality, one can use the word $1^{n} \hat{1}$.

Orbital/Schreier Graphs Many properties of automaton (semi)groups can be studied by exploring their so-called orbital (Schreier) graphs. For an $S$-automaton $\mathcal{T}=(Q, \Sigma, \delta)$ and a word $x \in \Sigma^{*} \cup \Sigma^{\omega}$ we denote the orbit of $x$ under the action of $\mathcal{T}$ by

$$
Q^{*} \circ x=\left\{q_{n} \ldots q_{1} \circ x \mid q_{1}, \ldots, q_{n} \in Q, n \in \mathbb{N}\right\} .
$$

On this set, we can define a natural graph structure: the orbital graph of $\mathcal{T}$ centered at $x$, denoted by $\mathcal{T} \circ x$, is a labeled directed graph with $Q^{*} \circ x$ as the node set and the edge set $\left\{y \stackrel{q}{\longrightarrow} q \circ x \mid y \in Q^{*} \circ x, q \in Q, q \circ\right.$ defined on $\left.y\right\}$. Notice that, for an $\omega$-word $\xi \in \Sigma^{\omega}$, the graph $\mathcal{T} \circ \xi$ is the limit in the sense of pointed Gromov-Hausdorff convergence of the sequence $\mathcal{T} \circ \xi_{n}$ where $\xi_{n}$ is the prefix of length $n$ of $\xi$. If $\mathcal{T}=(Q, \Sigma, \delta)$ is an $\bar{S}$-automaton (or even a $G$-automaton), then we can extend these notions to include inverses. Let $\overline{\mathcal{T}}=(\bar{Q}, \Sigma, \bar{\delta})$ be the inverse of $\mathcal{T}$, let $x \in \Sigma^{*} \cup \Sigma^{\omega}$ be a word, and let $\widetilde{Q}=Q \cup \bar{Q}$. Then, we can define the orbit of $x$ under the action of $\mathcal{T}$ as an $\bar{S}$-automaton:

$$
\widetilde{Q}^{*} \circ x=\left\{\widetilde{q}_{n} \ldots \widetilde{q}_{1} \circ x \mid \widetilde{q}_{1}, \ldots, \widetilde{q}_{n} \in \widetilde{Q}, n \in \mathbb{N}\right\} .
$$

Again, we have a natural graph structure on this set: the Schreier graph of $\mathcal{T}$ centered at $x$ is the labeled directed graph $\widetilde{\mathcal{T}} \circ x$ with node set $\widetilde{Q}^{*} \circ x$. It contains an edge $y \stackrel{\widetilde{q}}{\longrightarrow} z$ whenever $\widetilde{q} \circ y=z$ for $\widetilde{q} \in \widetilde{Q}$. Notice that $\mathcal{T} \circ x$ is always a sub-graph of $\widetilde{\mathcal{T}} \circ x$. Just like with $\mathcal{T} \circ \xi, \tilde{\mathcal{T}} \circ \xi$ is the limit (in the sense of pointed Gromov-Hausdorff convergence) of the sequence $\widetilde{\mathcal{T}} \circ \xi_{n}$ where $\xi_{n}$ is the prefix of length $n$ of $\xi$.

\footnotetext{
${ }^{8}$ This extension is inspired by [32, Fig. 8].
} 
Example 2.4. Recall the adding machine $\mathcal{T}$ from Example 2.3. The following figure depicts the infinite orbital graph $\mathcal{T} \circ 0^{\omega}$ (dark) and the additional vertices and edges in the Schreier graph $\widetilde{\mathcal{T}} \circ 0^{\omega}$ (light) schematically.

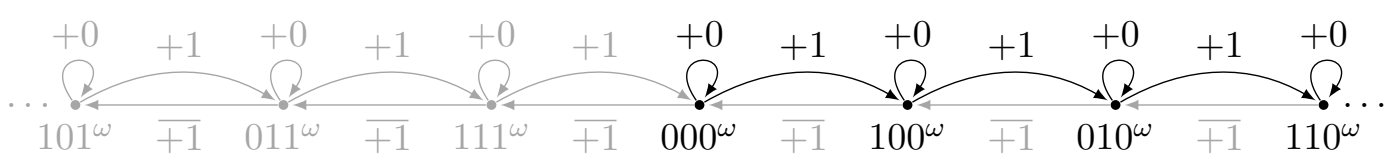

One may have noticed that $\widetilde{\mathcal{T}} \circ 0^{\omega}$ from Example 2.4 coincides with the Cayley graph of $\mathscr{G}(\mathcal{T})=(\mathbb{Z},+)$. This is not a coincidence but the reason why $\widetilde{\mathcal{T}} \circ x$ is called the Schreier graph: it is isomorphic to the coset graph of the stabilizer of $x$. For an $S$-automaton $\mathcal{T}=(Q, \Sigma, \delta)$ and a word $x \in \Sigma^{*} \cup \Sigma^{\omega}$, we define

$$
\operatorname{Stab}_{\mathcal{T}}(x)=\left\{\boldsymbol{q} \mid \boldsymbol{q} \in Q^{+}, \boldsymbol{q} \circ x=x\right\}
$$

as the set of (positive length) state sequences whose actions stabilize $x$. Accordingly, $\operatorname{Stab}_{\mathcal{T}}(x) \circ=\left\{\boldsymbol{q} \circ \mid \boldsymbol{q} \in Q^{+}, \boldsymbol{q} \circ x=x\right\} \subseteq \mathscr{S}(\mathcal{T})$ is the (semigroup) stabilizer of $x$ under the action induced by $\mathcal{T}$. Notice that, if $\mathcal{T}$ is an $\bar{S}$-automaton, then, with this notation, we have

$$
\operatorname{Stab}_{\mathcal{T} \sqcup \overline{\mathcal{T}}}(x)=\left\{\widetilde{\boldsymbol{q}} \circ \mid \widetilde{\boldsymbol{q}} \in(Q \sqcup \bar{Q})^{+}, \widetilde{\boldsymbol{q}} \circ x=x\right\} .
$$

If $\mathcal{T}$ is a $G$-automaton, then

$$
\operatorname{Stab}_{\mathcal{T} \sqcup \overline{\mathcal{T}}}(x) \circ=\left\{\widetilde{\boldsymbol{q}} \circ \mid \widetilde{\boldsymbol{q}} \in(Q \sqcup \bar{Q})^{*}, \widetilde{\boldsymbol{q}} \circ x=x\right\} \subseteq \mathscr{G}(\mathcal{T})
$$

is the group stabilizer of $x$ under the action induced by $\mathcal{T}$. The isomorphism between $\widetilde{\mathcal{T}} \circ x$ and $\mathscr{G} / \operatorname{Stab}_{\mathcal{T} \sqcup \overline{\mathcal{T}}}(x) \circ$ can now be seen easily (using the mapping $\boldsymbol{q} \circ \mapsto \boldsymbol{q} \operatorname{Stab}_{\mathcal{T} \sqcup \overline{\mathcal{T}}}(x) \circ$ ). In addition to the name, this connection has another consequence: the size of $\widetilde{\mathcal{T}} \circ x$ is the index of $\operatorname{Stab}_{\mathcal{T} \sqcup \overline{\mathcal{T}}}(x) \circ$ in $\mathscr{G}(\mathcal{T})$.

The finiteness of orbital and Schreier graphs is an important property in studying certain algebraic properties of the corresponding automaton structure. This relationship has been exploited in [15] in connection with the property of an automaton group to be free. In [15] the authors implicitly used the following fact for automaton groups. It states that, when considering automaton groups, it is indifferent whether one considers the orbital graph or the Schreier graphs. The former is finite if and only if the latter is.

Lemma 2.5. Let $\mathcal{T}=(Q, \Sigma, \delta)$ be a G-automaton and let $\overline{\mathcal{T}}=(\bar{Q}, \Sigma, \bar{\delta})$ be its inverse. Then, for any $x \in \Sigma^{*} \cup \Sigma^{\omega}$, we have

$$
Q^{*} \circ x=\widetilde{Q}^{*} \circ x \quad \text { or } \quad\left|Q^{*} \circ x\right|=\left|\widetilde{Q}^{*} \circ x\right|=\infty
$$

where $\widetilde{Q}=Q \sqcup \bar{Q}$.

Proof. Let $x \in \Sigma^{*} \cup \Sigma^{\omega}$ be arbitrary. Trivially, we have $Q^{*} \circ x \subseteq \widetilde{Q}^{*} \circ x$. Thus, it remains to show $\widetilde{Q}^{*} \circ x \subseteq Q^{*} \circ x$ if $Q^{*} \circ x$ is finite (which is always the case for $x \in \Sigma^{*}$ ). Suppose, $Q^{*} \circ x \subsetneq \widetilde{Q}^{*} \circ x$. Then, there is an element $y \in \widetilde{Q}^{*} \circ x \backslash Q^{*} \circ x$ such that $z \stackrel{\bar{q}}{\longrightarrow} y$ is an edge 
in $\tilde{\mathcal{T}} \circ x$ for some $z \in Q^{*} \circ x$ and some $\bar{q} \in \bar{Q}$. Because $Q^{*} \circ x$ is finite and because $\mathcal{T}$ is complete, $z$ must have an in-coming $q$-labeled edge from some $y^{\prime} \in Q^{*} \circ x$ by cardinality reasons. This yields the edge $z \stackrel{\bar{q}}{\longrightarrow} y^{\prime}$ in $\widetilde{\mathcal{T}} \circ x$. However, this implies $y=y^{\prime} \in Q^{*} \circ x$ (by the determinism of the graph); a contradiction.

In the next lemma, we show that the completeness of the automaton is essential in the statement of Lemma 2.5.

Lemma 2.6. Let $\mathcal{T}=(Q,\{0, \hat{0}, 1, \hat{1}\}, \delta)$ denote the following $\bar{S}$-automaton (a kind of "mark adding" variation of the adding machine from Example 2.3.

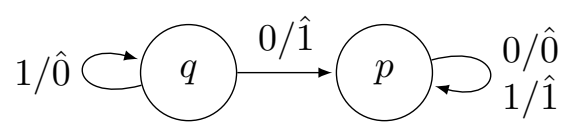

and let $\overline{\mathcal{T}}=(\bar{Q},\{0, \hat{0}, 1, \hat{1}\}, \bar{\delta})$ denote its inverse

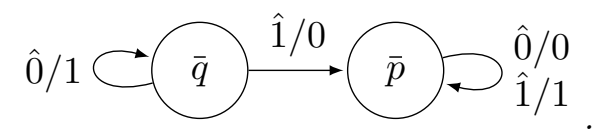

Then, we have that $\overline{\mathscr{S}}(\mathcal{T})$ is infinite and $\left|\widetilde{Q}^{*} \circ 0^{\omega}\right|=\infty$ where $\widetilde{Q}=Q \sqcup \bar{Q}$ but also that $\mathscr{S}(\mathcal{T})$ is finite and $\left|Q^{*} \circ \xi\right| \leq 2$ for all $\xi \in \Sigma^{\omega}$ and, in particular $\left|Q^{*} \circ 0^{\omega}\right|=2$.

Proof. Any $\omega$-word from $\{0,1\}^{\omega}$ containing only finitely many occurrences of 1 can be seen as the reverse binary representation of some natural number $n \in \mathbb{N}$ (with infinitely many leading/trailing zeros). For example, we have $0^{\omega}=\overleftarrow{\mathrm{bin}}(0)$ and $10110^{\omega}=\overleftarrow{\mathrm{bin}}(13)$, where $\overleftarrow{\operatorname{bin}}(n)$ denotes the (infinite) reverse binary representation of a natural number $n \in \mathbb{N}$.

Recall from Example 2.3, that the action of $q$ on such words basically increments the represented number by one. In the automaton depicted in the lemma, $q \circ$ additionally adds a decoration to each letter. Let $\langle\cdot\rangle:\{0,1\}^{*} \rightarrow\{\hat{0}, \hat{1}\}^{*}$ denote the isomorphism given by $\langle 0\rangle=\hat{0}$ and $\langle 1\rangle=\hat{1}$. Then, we have $q \circ \overleftarrow{\operatorname{bin}}(n)=\langle\overleftarrow{\operatorname{bin}}(n+1)\rangle$ and $p \circ=\langle\cdot\rangle$.

Because $p \circ$ and $q \circ$ are only defined on words over $\{0,1\}$ (and, in particular, not on words containing at least one $\hat{0}$ or $\hat{1}$ ), we have, for all $\eta \in \Sigma^{\omega}$, that $Q^{*} \circ \eta$ contains $\eta$ itself and, possibly, a single other word (if $\eta$ does not contain any $\hat{0}$ or $\hat{1}$ ). For example, we have $Q^{*} \circ 0^{\omega}=\left\{0^{\omega}, \hat{1} \hat{0}^{\omega}\right\}$. Additionally, this shows that $\mathscr{S}(\mathcal{T})$ is finite.

Since we can remove the decorations added by $q \circ$ using $\bar{p}$ (if we take the inverses into consideration), we have $\overleftarrow{\operatorname{bin}}(n)=(\bar{p} q)^{n} \circ 0^{\omega} \in \widetilde{Q}^{*} \circ 0^{\omega}$ for all $n \in \mathbb{N}$ and, thus, $\left|\widetilde{Q}^{*} \circ 0^{\omega}\right|=\infty$. This also shows that $\overline{\mathscr{S}}(\mathcal{T})$ is infinite.

Lemma 2.6 has another consequence. For a $G$-automaton $\mathcal{T}$, we have that $\mathscr{G}(\mathcal{T})$ is finite if and only if $\mathscr{S}(\mathcal{T})$ is [1. However, the lemma states that the analog for $\bar{S}$ automata does not hold: there is an $\bar{S}$-automaton $\mathcal{T}$ such that $\mathscr{S}(\mathcal{T})$ is finite but $\overline{\mathscr{S}}(\mathcal{T})$ is not. 


\section{Freeness, Positive Relations and the Dynamics in the Boundary}

In [15], the study of the algorithmic problem of checking whether an automaton group is free has been initiated. Formally, let FreEness be the decision problem:
Input:
a $G$-automaton $\mathcal{T}$
Question: is $\mathscr{G}(\mathcal{T})$ free?

One of the results from [15] on Freeness is a connection to the existence of finite Schreier graphs, which we recall in the next corollary.

Corollary 3.1. [15, Corollary 2] The algorithmic problem of establishing whether a group generated by a $G$-automaton $\mathcal{T}=(Q, \Sigma, \delta)$ is not free is equivalent to the problem of checking whether $\partial(\mathcal{T} \sqcup \overline{\mathcal{T}})$ possesses a finite orbital graph in the boundary centered at a periodic point $y^{\omega} \in(Q \sqcup \bar{Q})^{\omega}$ where $y$ is different to the identity in the free group $F G(Q)$. Furthermore, for a bi-reversible $G$-automaton $\mathcal{T}=(Q, \Sigma, \delta)$, checking if $\mathscr{G}(\mathcal{T})$ is free is equivalent to checking whether or not there exists a finite Schreier graph of $\partial(\mathcal{T} \sqcup \overline{\mathcal{T}})$ centered at an essentially non-trivial elemen $\bigsqcup^{9}$ from $(Q \sqcup \bar{Q})^{\omega}$.

Positive Relations. In the direction of Freeness, we may consider the weaker problem Positive Relations. For a given $G$-automaton $\mathcal{T}=(Q, \Sigma, \delta)$, the set of positive relations of $\mathscr{G}(\mathcal{T})$ with respect to $\mathcal{T}$ is given by

$$
\mathcal{P}(\mathcal{T})=\left\{\boldsymbol{q} \in Q^{+} \mid \boldsymbol{q}=\mathbb{1} \text { in } \mathscr{G}(\mathcal{T})\right\},
$$

i. e. it is the set of state sequences (which do not contain states from the inverse of $\mathcal{T}$ ) which act like the identity. So, for instance, if $\mathcal{P}(\mathcal{T})=\emptyset$, then the associated semigroup $\mathscr{S}(\mathcal{T})$ is torsion-free. This allows us to formally define the decision problem Positive RELATIONS:

\section{Input: $\quad$ a $G$-automaton $\mathcal{T}$}

Question: is $\mathcal{P}(\mathcal{T})=\emptyset$ ?

Notice that, in contrast to freeness, which is a property of the group, positive relations are dependent on the automaton representation. For example, if $\mathcal{T}$ denotes the adding machine from Example 2.3, then $\mathscr{G}(\mathcal{T})$ is the free group of rank one, which is obviously free, but the set $\mathcal{P}(\mathcal{T})$ of positive relations is not empty, since we have, for example, $Q \ni+0=\mathbb{1}$ in $\mathscr{G}(\mathcal{T})$. So, the presence of a sink state in a $G$-automaton will always cause the set of positive relations to be non-empty.

Despite its dependency on the presentation, studying positive relations is still worthwhile as, for example, the emptiness of the $\operatorname{set} \mathcal{P}(\mathcal{T})$ is strictly related to the dynamics of an automaton group $\mathscr{G}(\mathcal{T})$. While we refer the reader to [12] for further details, especially regarding reversible and bi-reversible automata, we give an example of this connection.

\footnotetext{
${ }^{9}$ For an $\omega$-word $\xi \in(Q \sqcup \bar{Q})^{\omega}$, consider the sequence of the prefixes of $\xi$ reduced in the free group $F G(Q)$. If this sequence contains a sub-sequence converging to an element from $(Q \sqcup \bar{Q})^{\omega}$ (and not to an element from $\left.(Q \sqcup \bar{Q})^{*}\right)$, then $\xi$ is essentially non-trivial. See [15, Proposition 4] for equivalent definitions of essentially trivial elements.
} 
In [12, Lemma 5.8], it is observed that, for a $G$-automaton $\mathcal{T}=(Q, \Sigma, \delta)$, the absence of positive relations $\mathcal{P}(\mathcal{T})=\emptyset$ implies the existence of a periodic point $u^{\omega}$, for some $u \in \Sigma^{*}$, with a non-trivial semigroup stabilizer $\operatorname{Stab}_{\mathcal{T}}\left(u^{\omega}\right) \circ \neq \emptyset$.

Another example of the connection is [15, Corollary 4]: if a reversible $G$-automaton $\mathcal{T}$ generates an infinite group $G$, then the index $\left[G: \operatorname{Stab}_{\mathcal{T} \sqcup \overline{\mathcal{T}}}\left(u^{\omega}\right) \circ\right]$ is infinite for all $u \in \Sigma^{*}$ if and only if the dual of $\mathcal{T}$ admits no positive relations: $\mathcal{P}(\partial \mathcal{T})=\emptyset$.

Positive Relations and the Structure of Orbital Graphs. Many interesting properties of automaton structures are reflected in the structure of their orbital graphs. For example, Proposition 4.1 in section 4 relates the finiteness of an automaton semigroup to the uniform boundedness of its orbital graphs. Similarly, the absence of positive relations in an automaton group is related to the absence of circles in some of its orbital graphs. We will develop this simple, yet interesting, connection next.

For any countable group $G$, we can identify each subgroup $H$ with its characteristic function, which maps an element $g \in G$ to 1 if $g \in H$ and to 0 , otherwise. In this way, we have identified the set $\operatorname{Sub}(G)$ of subgroups of $G$ with $\{0,1\}^{|G|}$, which we can in turn endow with the Tychonoff topology of the $|G|$-fold product of the discrete topological space $\{0,1\}$. For a $G$-automaton $\mathcal{T}=(Q, \Sigma, \delta)$, this allows us to consider the continuity of the map $\operatorname{Stab}_{\mathcal{T} \sqcup \overline{\mathcal{T}}}(\cdot) \circ: \Sigma^{\omega} \rightarrow \operatorname{Sub}(\mathscr{G}(\mathcal{T}))$ which maps $\xi$ to $\operatorname{Stab}_{\mathcal{T} \sqcup \overline{\mathcal{T}}}(\xi) \circ{ }^{10}$ We say an $\omega$-word $\xi \in \Sigma^{\omega}$ is singular if $\operatorname{Stab}_{\mathcal{T} \sqcup \overline{\mathcal{T}}}(\cdot) \circ$ is not continuous at $\xi$. In [12, Theorem 4.4] it is shown that, for every $G$-automaton, the set $\kappa$ of singular points has measure zero. Thus, if we denote by $\Theta=\Sigma^{\omega} \backslash \kappa$ the set of non-singular points, then $\Theta$ has measure one. Non-singular points can be characterized in the following way (see [12, Lemma 4.2]).

Lemma 3.2. Let $\mathcal{T}=(Q, \Sigma, \delta)$ be a $G$-automaton. An element $\xi \in \Sigma^{\omega}$ is not singular if and only if, for all $\widetilde{\boldsymbol{q}} \in \operatorname{Stab}_{\mathcal{T} \sqcup \overline{\mathcal{T}}}(\xi)$, there exists a prefix $u \in \Sigma^{*}$ of $\xi$ such that $\widetilde{\boldsymbol{q}} \cdot u \circ=\mathbb{1}$.

Using this characterization, we can prove the following result on the set of non-singular points.

Lemma 3.3. For every $G$-automaton $\mathcal{T}=(Q, \Sigma, \delta)$, the set $\Theta$ of non-singular points is $\mathscr{G}(\mathcal{T})$-stable, i. e. we have $\widetilde{\boldsymbol{q}} \circ \xi \in \Theta$ for all $\xi \in \Theta$ and all $\widetilde{\boldsymbol{q}} \in(Q \sqcup \bar{Q})^{*}$, where $\bar{Q}$ is the state set of $\overline{\mathcal{T}}$.

Proof. Let $\xi \in \Theta$ and $\widetilde{\boldsymbol{q}} \in(Q \sqcup \bar{Q})^{*}$ and define $\eta=\widetilde{\boldsymbol{q}} \circ \xi$. We need to show $\eta \in \Theta$. For any $\widetilde{\boldsymbol{p}} \in \operatorname{Stab}_{\mathcal{T} \sqcup \overline{\mathcal{T}}}(\eta)$, we have $\widetilde{\boldsymbol{q}}^{-1} \widetilde{\boldsymbol{p}} \widetilde{\boldsymbol{q}} \in \operatorname{Stab}_{\mathcal{T} \cup \overline{\mathcal{T}}}(\xi)$. Thus, by the characterization in Lemma 3.2, there is a prefix $u$ of $\xi$ such that $\widetilde{\boldsymbol{q}}^{-1} \widetilde{\boldsymbol{p}} \widetilde{\boldsymbol{q}} \cdot u \circ=\mathbb{1}$. Hence, $v=\widetilde{\boldsymbol{q}} \circ u$ is a prefix of $\eta$ and we have $\widetilde{\boldsymbol{p}} \in \operatorname{Stab}_{\mathcal{T} \sqcup \overline{\mathcal{T}}}(v)$. Now, for $\widetilde{\boldsymbol{r}}=\widetilde{\boldsymbol{q}} \cdot u$, we have

$$
\begin{aligned}
\widetilde{\boldsymbol{q}}^{-1} \widetilde{\boldsymbol{p}} \widetilde{\boldsymbol{q}} \cdot u & =\left[\widetilde{\boldsymbol{q}}^{-1} \widetilde{\boldsymbol{p}} \cdot(\widetilde{\boldsymbol{q}} \circ u)\right][\widetilde{\boldsymbol{q}} \cdot u]=\left[\widetilde{\boldsymbol{q}}^{-1} \widetilde{\boldsymbol{p}} \cdot v\right][\widetilde{\boldsymbol{q}} \cdot u]=\left[\widetilde{\boldsymbol{q}}^{-1} \cdot(\widetilde{\boldsymbol{p}} \circ v)\right][\widetilde{\boldsymbol{p}} \cdot v][\widetilde{\boldsymbol{q}} \cdot u] \\
& =\left[\widetilde{\boldsymbol{q}}^{-1} \cdot v\right][\widetilde{\boldsymbol{p}} \cdot v][\widetilde{\boldsymbol{q}} \cdot u]=\boldsymbol{r}^{-1}[\widetilde{\boldsymbol{p}} \cdot v] \boldsymbol{r}
\end{aligned}
$$

for the last step, notice the equality $(\widetilde{\boldsymbol{q}} \cdot u)^{-1}=\widetilde{\boldsymbol{q}}^{-1} \cdot v$. From $\mathbb{1}=\widetilde{\boldsymbol{q}}^{-1} \widetilde{\boldsymbol{p}} \widetilde{\boldsymbol{q}} \cdot u \circ$, thus, also follows $\widetilde{\boldsymbol{p}} \cdot v \circ=\mathbb{1}$. Since we have chosen $\widetilde{\boldsymbol{p}}$ arbitrarily from $\operatorname{Stab}_{\mathcal{T} \sqcup \overline{\mathcal{T}}}(\eta)$, we have $\eta \in \Theta$ by Lemma 3.2 .

\footnotetext{
${ }^{10}$ For the special case of Grigorchuk's group, the map was studied by Vorobets 39. Here, we consider the more general case of arbitrary automaton groups.
} 
Now, we are prepared to characterize the absence of positive relations in terms of orbital graphs.

Proposition 3.4. For every $G$-automaton $\mathcal{T}=(Q, \Sigma, \delta)$, the following are equivalent:

(i) There is a non-singular element $\xi \in \Sigma^{\omega}$ such that $\mathcal{T} \circ \xi$ contains a (non-trivial) cycle;

(ii) $\mathcal{P}(\mathcal{T}) \neq \emptyset$;

Proof. (ii) $\Longrightarrow$ (i). If $\boldsymbol{q} \in \mathcal{P}(\mathcal{T}) \neq \emptyset$, then $\mathcal{T} \circ \xi$ contains the circle $\xi \stackrel{\boldsymbol{q}}{\longrightarrow} \xi$ for every $\xi \in \Sigma^{\omega}$. So, in particular, it contains a cycle for every non-singular $\xi$.

(i) $\Longrightarrow$ (ii). Suppose that $\mathcal{T} \circ \eta$ for some non-singular $\eta \in \Sigma^{\omega}$ contains a cycle $\xi \stackrel{p}{\longrightarrow} \xi$ at a node $\xi \in Q^{*} \circ \eta$ labeled by $\boldsymbol{p} \in Q^{+}$. We have $\xi=\boldsymbol{q} \circ \eta$ for some $\boldsymbol{q} \in Q^{*}$ and, thus, by Lemma 3.3, $\xi$ is also non-singular. Therefore, by Lemma 3.2, there is prefix $u \in \Sigma^{*}$ of $\xi$ such that $\boldsymbol{q} \cdot u=\mathbb{1}$, i. e. we have $\boldsymbol{q} \cdot u \in \mathcal{P}(\mathcal{T}) \neq \emptyset$.

Undecidability of Positive Relations In [12, it was left open whether or not Positive Relations is undecidable. In this section, we will prove undecidability of Positive RELATIONS and the freeness problem for automaton semigroups. Crucial to our proof is a construction by Sunić and Ventura [38 ${ }^{11}$. Any pair of a $d \times d$ matrix $M$ and a vector $\boldsymbol{v} \in \mathbb{Z}^{d}$ gives rise to an affine transformation

$$
\begin{aligned}
M_{\boldsymbol{v}}: \mathbb{Z}^{d} & \rightarrow \mathbb{Z}^{d} \\
\boldsymbol{u} & \mapsto \boldsymbol{v}+M \boldsymbol{u} .
\end{aligned}
$$

We denote by $\mathrm{SAff}_{d}(\mathbb{Z})$ the semigroup of all these affine transformations. Since the affine transformation $M_{\boldsymbol{v}}$ is invertible if and only if the matrix $M$ is, we can also define $\operatorname{Aff}_{d}(\mathbb{Z})$, the group of affine transformations of $\mathbb{Z}^{d}$.

Although Sunić and Ventura only considered invertible matrices, their general construction also yields the following lemma, which considers non-invertible matrices.

Lemma 3.5. Let $\mathcal{M}$ be a finite set of $\mathbb{Z}^{d \times d}$ matrices. Furthermore, for every $M \in \mathcal{M}$, let $V_{M}$ be the finite set of vectors $\boldsymbol{v} \in \mathbb{Z}^{d}$ such that all components of $\boldsymbol{v}$ are between $-\|M\|$ and $\|M\|-1$. Here, $\|M\|$ denotes the norm $\|M\|=\max _{1 \leq i \leq d} \sum_{j=1}^{d}\left|m_{i, j}\right|$ where $m_{i, j}$ is the entry in the $i^{\text {th }}$ row and $j^{\text {th }}$ column of $M$.

Then, one can compute a complete $S$-automaton $\mathcal{T}_{\mathcal{M}}$ with state set

$$
Q_{\mathcal{M}}=\left\{m_{M, \boldsymbol{v}} \mid M \in \mathcal{M}, \boldsymbol{v} \in V_{M}\right\}
$$

such that the homomorphism $\varphi: \mathscr{S}\left(\mathcal{T}_{\mathcal{M}}\right) \rightarrow \operatorname{SAff}_{d}(\mathbb{Z})$ induced by $\varphi\left(m_{M, \boldsymbol{v}}\right)=M_{\boldsymbol{v}}$ for $M \in \mathcal{M}$ and $\boldsymbol{v} \in V_{M}$ is a well-defined isomorphism from $\mathscr{S}\left(\mathcal{T}_{\mathcal{M}}\right)$ into the subsemigroup of $\mathrm{SAff}_{d}(\mathbb{Z})$ generated by $\left\{M_{\boldsymbol{v}} \mid M \in \mathcal{M}, \boldsymbol{v} \in V_{M}\right\}$.

If all matrices in $\mathcal{M}$ are invertible, then $\mathcal{T}_{\mathcal{M}}$ is inverse-deterministic and $\varphi$ extends to a well-defined isomorphism from $\mathscr{G}(\mathcal{T})$ into the subgroup of $\mathrm{Aff}_{d}(\mathbb{Z})$ generated by $\left\{M_{\boldsymbol{v}} \mid\right.$ $\left.M \in \mathcal{M}, \boldsymbol{v} \in V_{M}\right\}$.

For the

\footnotetext{
${ }^{11}$ In fact, it is a rediscovery of a result by Brunner and Sidki 8 .
} 
Proof. The proof is based on the construction given in [38, which uses $n$-adic integers. The ring $\mathbb{Z}_{n}$ of $n$-adic integers is the projective limit of the rings $\mathbb{Z} / n^{k} \mathbb{Z}$. Its elements are right-infinite sequences $\left(a_{k}\right)_{k \in \mathbb{Z}_{+}}$such that, for every $k, a_{k}$ is in $\mathbb{Z} / n^{k} \mathbb{Z}$ and $a_{k} \equiv a_{l} \bmod$ $n^{k}$ for all $l \geq k$. In this representation, multiplication and addition are component-wise operations and a (normal) integer $z \in \mathbb{Z}$ is a sequence which becomes stationary with value $z$.

Another way of representing $n$-adic integers is by their $n$-adic expansion. Any (formal) sum $Z=\sum_{k=0}^{\infty} d_{k} n^{k}$ where the coefficients $d_{k}$ are from the range $0 \leq d_{k} \leq n-1$ represents an $n$-adic integer $\left(Z \bmod n, Z \bmod n^{2}, Z \bmod n^{3}, \ldots\right)=\left(d_{0}, d_{0}+d_{1} \cdot n, d_{0}+\right.$ $\left.d_{1} \cdot n+d_{2} \cdot n^{2}, \ldots\right)$. On the other hand, any $n$-adic integer $Z=\left(a_{1}, a_{2}, a_{3}, \ldots\right)$ can be written as a sum $\sum_{k=0}^{\infty} d_{k} n^{k}$ with $d_{k}=\frac{a_{k+1}-a_{k}}{n^{k}}$ (where we set $a_{0}=0$ ). Notice that $a_{k+1}-a_{k} \equiv 0 \bmod n^{k}$ and $a_{k+1}<n^{k+1}$. The $n$-adic expansion is then the $\omega$-word $d_{0} d_{1} d_{2} \ldots$

By using $n$-adic expansions, any vector $\boldsymbol{z} \in \mathbb{Z}_{n}^{d}$ can be represented by $d$ many $\omega$-words over the alphabet $Y_{n}=\{0, \ldots, n-1\}$ or by one $\omega$-word over the alphabet $X_{n}=Y_{n}^{d}=$ $\{0, \ldots, n-1\}^{d}$. The latter is the encoding on which the automata constructed in [38] act.

By [38, Lemma 4.5], there is a $G$-automaton $\mathcal{A}_{M, n}$ with state set $\left\{m_{\boldsymbol{v}} \mid \boldsymbol{v} \in V_{M}\right\}$ for every invertible $\mathbb{Z}^{d \times d}$ matrix $M$ such that, for every vector $\boldsymbol{v} \in V_{m} \subseteq \mathbb{Z}^{d} \subsetneq \mathbb{Z}_{n}^{d}$, the state $m_{\boldsymbol{v}}$ acts like $M_{\boldsymbol{v}}$ extended into an affine transformation $M_{\boldsymbol{v}}: \mathbb{Z}_{n}^{d} \rightarrow \mathbb{Z}_{n}^{d}$ with $M_{\boldsymbol{v}}(\boldsymbol{w})=\boldsymbol{v}+M \boldsymbol{w}$. Here, $n$ is a number relatively prime to the (non-zero) determinant of $M$ to preserve invertibility of $M$ over the $n$-adic integers. The described construction of the automaton is clearly computable. Furthermore, it does not depend on the invertibility of the matrix $M$; if the matrix is not invertible, the obtained automaton is not necessarily a $G$-automaton anymore, but a complete $S$-automaton whose states still act in the way described above.

As the automaton $\mathcal{T}_{\mathcal{M}}$, we can choose the disjoint union $\bigsqcup_{M \in \mathcal{M}} \mathcal{A}_{M, n}$ for some $n$ which is coprime to all non-zero determinants of the matrices in $\mathcal{M}$.

The semigroup part of the lemma's assertion follows if we choose $\varphi$ as the restriction of maps $\mathbb{Z}_{n}^{d} \rightarrow \mathbb{Z}_{n}^{d}$ over $n$-adic integer vectors to maps $\mathbb{Z}^{d} \rightarrow \mathbb{Z}^{d}$ of (normal) integer vectors. The only missing part, here, is to show injectivity (see also [38, Lemma 4.1]). For this, assume that, for some matrices $M, M^{\prime} \in \mathbb{Z}^{d \times d}$ and some vectors $\boldsymbol{v}, \boldsymbol{v}^{\prime} \in \mathbb{Z}^{d}$, $M_{\boldsymbol{v}}(\boldsymbol{u})=M_{\boldsymbol{v}^{\prime}}^{\prime}(\boldsymbol{u})$ holds for all $\boldsymbol{u} \in \mathbb{Z}^{d}$ but, for some vector $\boldsymbol{w} \in \mathbb{Z}_{n}^{d} \backslash \mathbb{Z}^{d}$, we have $M_{\boldsymbol{v}}(\boldsymbol{w}) \neq M_{\boldsymbol{v}^{\prime}}^{\prime}(\boldsymbol{w})$. Choosing $\boldsymbol{u}=\mathbf{0}$ as the zero vector yields $\boldsymbol{v}=\boldsymbol{v}^{\prime}$. Thus, there is some $i \in\{1, \ldots, d\}$ such that $\sum_{j=1}^{d} m_{i, j} w_{j} \neq \sum_{j=1}^{d} m_{i, j}^{\prime} w_{j}$ where $m_{i, 1}, \ldots, m_{i, d} \in \mathbb{Z}$ and $m_{i, 1}^{\prime}, \ldots, m_{i, d}^{\prime} \in \mathbb{Z}$ are the entries of the $i^{\text {th }}$ row of $M$ and $M^{\prime}$, respectively, and $w_{1}, \ldots, w_{d} \in \mathbb{Z}_{n}$ are the components of $\boldsymbol{w}$. As an $n$-adic integer, we can write each $w_{j}$ as a sequence $\left(a_{j, 1}, a_{j, 2}, \ldots\right)$ with $a_{j, k} \in \mathbb{Z} / n^{k} \mathbb{Z}$. As addition and multiplication in $\mathbb{Z}_{n}$ are component-wise in this representation, there is some $k \in \mathbb{Z}_{+}$with

$$
\left[\sum_{j=1}^{d} m_{i, j} a_{j, k}\right] \bmod n^{k} \neq\left[\sum_{j=1}^{d} m_{i, j}^{\prime} a_{j, k}\right] \bmod n^{k},
$$


which implies $\sum_{j=1}^{d} m_{i, j} a_{j, k} \neq \sum_{j=1}^{d} m_{i, j}^{\prime} a_{j, k}$. Thus, we have $M_{\boldsymbol{v}}(\boldsymbol{u}) \neq M_{\boldsymbol{v}^{\prime}}^{\prime}(\boldsymbol{u})$ if we choose $\boldsymbol{u} \in \mathbb{Z}^{d}$ in such a way that the $j^{\text {th }}$ component is equal to $a_{j, k}$; this constitutes a contradiction.

It turns out that the relations in the (semi)group generated by $\mathcal{T}_{\mathcal{M}}$ are closely related to those in the linear (semi)group generated by the matrices in $\mathcal{M}$. We state and prove this connection in the next lemma.

Lemma 3.6. Let $\mathcal{M}$ be a finite set of $\mathbb{Z}^{d \times d}$ matrices and let $\mathcal{T}_{\mathcal{M}}$ be the automaton from Lemma 3.5. Then, for any sequence $M_{1} \ldots M_{k}$ and $N_{1} \ldots N_{l}$ of matrices from $\mathcal{M}$, we have

$$
M_{1} \ldots M_{k}=N_{1} \ldots N_{l} \Longleftrightarrow m_{M_{1}, \boldsymbol{v}_{1}} \ldots m_{M_{k}, \boldsymbol{v}_{k}}=m_{N_{1}, \boldsymbol{w}_{1}} \ldots m_{N_{l}, \boldsymbol{w}_{l}}
$$

for some vectors $\boldsymbol{v}_{i}$ and $\boldsymbol{w}_{j}$ such that all $m_{M_{i}, \boldsymbol{v}_{i}}$ and $m_{N_{j}, \boldsymbol{w}_{j}}$ are states in $Q_{\mathcal{M}}$.

In particular, if we denote by $I$ the $d \times d$ identity matrix, we have

$$
M_{1} \ldots M_{k}=I \Longleftrightarrow m_{M_{1}, \boldsymbol{v}_{1}} \ldots m_{M_{k}, \boldsymbol{v}_{k}} \text { acts like the identity }
$$

for some vectors $\boldsymbol{v}_{i}$ such that all $m_{M_{i}, \boldsymbol{v}_{i}}$ are states in $Q_{\mathcal{M}}$.

Proof. The second part of the lemma follows from the first one by choosing $l=0$. The direction from left to right of the first part follows by choosing all vectors $\boldsymbol{v}_{1}, \ldots, \boldsymbol{v}_{k}$ and $\boldsymbol{w}_{1}, \ldots, \boldsymbol{w}_{l}$ as the $d$-dimensional zero vector $\mathbf{0}$ as, then, we have

$$
\varphi\left(m_{M_{1}, \mathbf{0}} \ldots m_{M_{k}, \mathbf{0}}\right) \boldsymbol{u}=M_{1} \ldots M_{k} \boldsymbol{u}=N_{1} \ldots N_{l} \boldsymbol{u}=\varphi\left(m_{N_{1}, \mathbf{0}} \ldots m_{N_{l}, \mathbf{0}}\right) \boldsymbol{u}
$$

for all $\boldsymbol{u} \in \mathbb{Z}^{d}$ where $\varphi$ is the isomorphism from Lemma 3.5.

For the other direction, suppose we have $m_{M_{1}, \boldsymbol{v}_{1}} \ldots m_{M_{k}, \boldsymbol{v}_{k}}=m_{N_{1}, \boldsymbol{w}_{1}} \ldots m_{N_{l}, \boldsymbol{w}_{l}}$ for some vectors ${ }^{12} \boldsymbol{v}_{i}$ and $\boldsymbol{w}_{j}$. Because the images under the isomorphism $\varphi$ must be equal as well, we have

$$
\left(M_{1}\right)_{\boldsymbol{v}_{1}} \ldots\left(M_{k}\right)_{\boldsymbol{v}_{k}}(\boldsymbol{u})=\left(N_{1}\right)_{\boldsymbol{w}_{1}} \ldots\left(N_{l}\right)_{\boldsymbol{w}_{l}}(\boldsymbol{u})
$$

for all $\boldsymbol{u} \in \mathbb{Z}^{d}$. Since both sides are affine transformations, there are vectors $\boldsymbol{v}, \boldsymbol{w} \in \mathbb{Z}^{d}$ such that

$$
\boldsymbol{v}+M_{1} \ldots M_{k} \boldsymbol{u}=\left(M_{1}\right)_{\boldsymbol{v}_{1}} \ldots\left(M_{k}\right)_{\boldsymbol{v}_{k}}(\boldsymbol{u})=\left(N_{1}\right)_{\boldsymbol{w}_{1}} \ldots\left(N_{l}\right)_{\boldsymbol{w}_{l}}(\boldsymbol{u})=\boldsymbol{w}+N_{1} \ldots N_{l} \boldsymbol{u}
$$

holds for all $\boldsymbol{u} \in \mathbb{Z}^{d}$. Setting $\boldsymbol{u}=\mathbf{0}$, we obtain $\boldsymbol{v}=\boldsymbol{w}$ and, thus, $M_{1} \ldots M_{k} \boldsymbol{u}=N_{1} \ldots N_{l} \boldsymbol{u}$ for all $\boldsymbol{u} \in \mathbb{Z}^{d}$.

We are now prepared to prove undecidability of Positive Relations.

Theorem 3.7. Positive Relations is undecidable.

\footnotetext{
${ }^{12}$ Indeed, for this direction of the proof, we do not require the vectors to come from $V_{M_{i}}$ or $V_{N_{j}}$, respectively.
} 
Proof. We reduce the Identity Correspondence Problem (ICP) [4] to Positive RelaTIONS. It is the following decision problem. Let $F G(\Sigma)$ denote the free group over an alphabet $\Sigma$.

Constant: a binary alphabet $\Sigma=\{a, b\}$

Input: $\quad m \in \mathbb{N}, \Pi=\left\{\left(s_{1}, t_{1}\right),\left(s_{2}, t_{2}\right), \ldots,\left(s_{m}, t_{m}\right)\right\} \subseteq F G(\Sigma) \times F G(\Sigma)$

Question: is there a finite sequence $l_{1}, l_{2}, \ldots, l_{k}$ of indices with $k \geq 1$ where

$1 \leq l_{i} \leq m$ for $i=1, \ldots, k$ such that

$$
s_{l_{1}} s_{l_{2}} \ldots s_{l_{k}}=t_{l_{1}} t_{l_{2}} \ldots t_{l_{k}}=\varepsilon
$$

holds, where $\varepsilon$ is the empty word?

Since ICP is undecidable ${ }^{13}$ [4, Theorem 11], this reduction proves the undecidability of Positive Relations.

We consider the usual group embedding $\rho$ of $F G(\Sigma)$ into $S L_{2}(\mathbb{Z})$ defined on $\Sigma$ by

$$
\rho(a)=\left(\begin{array}{ll}
1 & 2 \\
0 & 1
\end{array}\right), \rho(b)=\left(\begin{array}{ll}
1 & 0 \\
2 & 1
\end{array}\right), \rho\left(a^{-1}\right)=\left(\begin{array}{cc}
1 & -2 \\
0 & 1
\end{array}\right), \rho\left(b^{-1}\right)=\left(\begin{array}{cc}
1 & 0 \\
-2 & 1
\end{array}\right)
$$

and use the same encoding of pairs from $F G(\Sigma) \times F G(\Sigma)$ as in [4, Theorem 13]: for each pair $\left(s_{i}, t_{i}\right) \in \Pi$, we define the block matrix

$$
H_{i}=\left(\begin{array}{cc}
\rho\left(s_{i}\right) & O_{2} \\
O_{2} & \rho\left(t_{i}\right)
\end{array}\right)
$$

where $O_{2}$ is the $2 \times 2$ zero-matrix. Let $\mathcal{H}$ be the set of these $4 \times 4$ invertible integer matrices $\left\{H_{1}, H_{2}, \ldots, H_{m}\right\}$. Note that existence of a solution $l_{1}, l_{2}, \ldots, l_{k}$ for ICP is equivalent to having

$$
H_{l_{1}} H_{l_{2}} \ldots H_{l_{k}}=I
$$

where $I$ is the $4 \times 4$ identity matrix.

Therefore, we have so far reduced an instance of ICP to the problem of checking whether the matrix semigroup generated by a finite set $\mathcal{H}$ of invertible integer matrices contains the identity. To reduce this problem to Positive Relations, we use the $G$-automaton $\mathcal{T}_{\mathcal{H}}$ from Lemma 3.5. As the automaton is computable, it remains to show that $\mathcal{P}\left(\mathcal{T}_{\mathcal{H}}\right)$ is non-empty if and only if the linear semigroup generated by $\mathcal{H}$ contains the identity matrix. This, however, is basically the second part of Lemma 3.6.

From Theorem 3.7 and Proposition 3.4 we immediately obtain the following corollary.

Corollary 3.8. Let $\mathcal{T}=(Q, \Sigma, \delta)$ be a $G$-automaton. The algorithmic problem of checking whether any of the orbital graphs $\mathcal{T} \circ \xi$ centered at a non-singular element $\xi \in \Sigma^{\omega}$ contains a cycle is undecidable.

In the proof of Theorem 3.7, we have reduced ICP to Positive Relations. However, we can use the idea of this proof also for another reduction. Re-consider (GRouP)

\footnotetext{
${ }^{13}$ In fact, it is already undecidable for constant $m$ with $m=8(n-1)$, where $n$ is the minimal number of pairs for which the Restricted $P C P$ is undecidable
}

The rest of this section is flawed; see the erratum below. 
FreEness, the freeness problem for automaton groups:

Input: a $G$-automaton $\mathcal{T}$

Question: is $\mathscr{G}(\mathcal{T})$ free?

If we make some straightforwards modifications to Lemma 3.6 to also cover inverses (or arbitrary powers of the matrices and states in general), then we also obtain a reduction from the following variant of $\mathbf{I C P}$, which we will call the Inverse Identity Correspondence Problem (IICP) to GRoup FreEness.

Constant: a binary alphabet $\Sigma=\{a, b\}$

Input: $\quad m \in \mathbb{N}, \Pi=\left\{\left(s_{1}, t_{1}\right),\left(s_{2}, t_{2}\right), \ldots,\left(s_{m}, t_{m}\right)\right\} \subseteq F G(\Sigma) \times F G(\Sigma)$

Question: is there a finite sequence $l_{1}, l_{2}, \ldots, l_{k}$ of indices with $k \geq 1$ where $1 \leq l_{i} \leq m$ for $i=1,2, \ldots, k$, and $e_{1}, e_{2} \ldots, e_{k} \in\{1,-1\}$ with $l_{i} \neq l_{i+1}$ or $e_{i}=e_{i+1}$ for $i=1, \ldots, k-1$ such that

$$
s_{l_{1}}^{e_{1}} s_{l_{2}}^{e_{2}} \ldots s_{l_{k}}^{e_{k}}=t_{l_{1}}^{e_{1}} t_{l_{2}}^{e_{2}} \ldots t_{l_{k}}^{e_{k}}=\varepsilon
$$

holds, where $\varepsilon$ is the empty word?

We state this result in the following proposition.

Proposition 3.9. IICP is reducible to Group Freeness. In particular, if IICP is undecidable, then so is Group Freeness.

This raises an obvious question:

Open Problem 3.10. Is IICP decidable?

Another problem related to the proof of Theorem 3.7 is the following.

Open Problem 3.11. The automata devised in [8, 38] and used in Lemma 3.5 are not reversible. Are there also (bi-)reversible $G$-automata generating these groups?

This problem is interesting because a positive answer would lead to undecidability of the decision problem whether all Schreier graphs cantered at periodic $\omega$-words of a $G$ automaton are infinite:

Input: $\quad$ a (bi-)reversible $G$-automaton $\mathcal{T}=(Q, \Sigma, \delta)$

Question: is $\widetilde{Q}^{*} \circ u^{\omega}$ infinite for all finite words $u \in \Sigma^{+}$(where $\widetilde{Q}$ is the union of the states of $\mathcal{T}$ and its inverse)?

The reduction is based on the fact that $\partial \mathcal{T}=\emptyset$ holds if and only if the stabilizer $\operatorname{Stab}_{\mathcal{T} \sqcup \widetilde{\mathcal{T}}}\left(u^{\omega}\right) \circ$ has infinite index in $\mathscr{G}(\mathcal{T})$ for all $u \in \Sigma^{+}[15$, Corollary 4]. So, one can reduce a strengthened version of Positive Relations where the input automaton is also (bi-)reversible to the above problem by taking the dual. This strengthened version is undecidable by the same proof as for Theorem 3.7 if one can compute (bi-)reversible $G$-automata for Lemma 3.5. 
Freeness Problem for Automaton Semigroups. In the direction of the freeness problems for automaton structures, we may also consider a strengthened version of the freeness problem for automaton semigroups: $G$-SEMIGRoup FrEENESS. It is the following problem.

\section{Input: $\quad$ a $G$-automaton $\mathcal{T}$}

Question: is $\mathscr{S}(\mathcal{T})$ free?

Besides its obvious connection to the freeness problem of automaton groups and semigroups, this problem is also interesting since, in literature, $G$-automata quite often generate free semigroups, see for instance [13, 14, 15, 33, 37]. In [13, Theorem 3.2], for example, decidable sufficient conditions for reset ${ }^{14} G$-automata to generate free semigroups are presented. Moreover, it is a well known fact that groups containing free semigroups (in at least two generators) have exponential growth. Usually, it seems considerably more easy to show that an automaton generates a free semigroup than to show that it generates a free group. However, it seems to be easier to show undecidability of the freeness problem for automaton semigroups than to study the freeness problem for automaton groups as the following theorem demonstrates.

Theorem 3.12. G-Semigroup Freeness is undecidable.

Proof. Consider the Matrix Semigroup Freeness problem

Input: $\quad$ a finite non-empty set $\mathcal{M}$ of invertible matrices from $\mathbb{N}^{3 \times 3}$

Question: is the linear semigroup generated by $\mathcal{M}$ free?

that has been proven undecidable in [27]. As in the proof of Theorem 3.7, we can use the $G$-automaton $\mathcal{T}_{\mathcal{M}}$ from Lemma 3.5 to reduce MAtrix Semigroup Freeness to $G$-Semigroup Freeness. By Lemma 3.6, the semigroup generated by $\mathcal{T}_{\mathcal{M}}$ is free if and only if so is the linear semigroup generated by $\mathcal{M}$.

As $G$-Semigroup Freeness is a strengthened version of the freeness problem for automaton semigroups, we get the following corollary, which solves an open problem of Grigorchuk, Nekrashevych and Sushchansky [23, 7.2 b)].

Corollary 3.13. The freeness problem for automaton semigroups

Input: $\quad$ an $S$-automaton $\mathcal{T}$

Question: is $\mathscr{S}(\mathcal{T})$ free?

is undecidable.

Theorem 3.12 can also be interpreted outside the context of automaton structures. Remember that any $S$-automaton $\mathcal{T}=(Q, \Sigma, \delta)$ is, by definition, a synchronous, deterministic finite state transducer whose input and output alphabets coincide. In a more automata theoretic setting, one would not use these transducers to define semigroups but to define (rational) relations. For this, we need to select an initial state $q_{0} \in Q$ and a set of final states $F \subseteq Q$. Under this choice, the rational relation accepted by $\mathcal{T}$ is

$$
\mathcal{R}\left[\mathcal{T}, q_{0}, F\right]=\left\{(u, v) \in \Sigma^{*} \times \Sigma^{*} \mid q_{0} \circ u=v, q_{0} \cdot u \in F\right\},
$$

\footnotetext{
${ }^{14}$ An $S$-automaton $\mathcal{T}=(Q, \Sigma, \delta)$ is called a reset $S$-automaton if there is a finite word $u \in \Sigma^{*}$ and a state $q_{0} \in Q$ with $q \cdot u=q_{0}$ for all $q \in Q$.
} 
i. e. a pair of finite words $(u, v)$ is in the relation if and only if, when reading $u$ starting in $q_{0}$, one ends in a state belonging to $F$ and the output is $v$.

Now, Theorem 3.12 yields the following undecidability result:

Corollary 3.14. The problem ${ }^{15}$

Input: a synchronous, complete, deterministic, invertible, finite-state transducer $\mathcal{T}$ with coinciding input and output alphabet and state set $Q$, in which every state is final

Question: is there a $k \geq 1$ such that, in the $k^{\text {th }}$-power of $\mathcal{T}$, one can choose two different initial states $\boldsymbol{p}, \boldsymbol{q} \in Q^{k}$ with $\mathcal{R}\left[\mathcal{T}^{k}, \boldsymbol{p}, Q^{k}\right]=\mathcal{R}\left[\mathcal{T}^{k}, \boldsymbol{q}, Q^{k}\right]$ ?

is undecidable.

Proof. The reduction function from (the complement of) $G$-Semigroup Freeness to this problem is the identity function with one exception: if the input automaton contains only one state, then it generates a finite and, thus, non-free semigroup; therefore, the reduction function can map these automata to an arbitrary but fixed automaton with a positive answer to the above question.

We need to show that, for a $G$-automaton $\mathcal{T}=(Q, \Sigma, \delta)$ with at least two states, the semigroup $\mathscr{S}(\mathcal{T})$ is not free if and only if there is a $k \geq 1$ and $\boldsymbol{p}, \boldsymbol{q} \in Q^{k}$ with $\boldsymbol{p} \neq \boldsymbol{q}$ such that $\mathcal{R}\left[\mathcal{T}^{k}, \boldsymbol{p}, Q^{k}\right]=\mathcal{R}\left[\mathcal{T}^{k}, \boldsymbol{q}, Q^{k}\right]$. Notice that we have $\boldsymbol{p} \circ=\boldsymbol{q} \circ$ if and only if we have $\mathcal{R}\left[\mathcal{T}^{|\boldsymbol{p}|}, \boldsymbol{p}, Q^{|\boldsymbol{p}|}\right]=\mathcal{R}\left[\mathcal{T}^{|\boldsymbol{q}|}, \boldsymbol{q}, Q^{|\boldsymbol{q}|}\right]$ for any par $\boldsymbol{p}, \boldsymbol{q} \in Q^{+}$. Thus, it suffices to show that there are $\boldsymbol{p}, \boldsymbol{q} \in Q^{+}$with $\boldsymbol{p} \neq \boldsymbol{q}$ but $|\boldsymbol{p}|=|\boldsymbol{q}|$ and $\boldsymbol{p} \circ=\boldsymbol{q} \circ$ if $\mathscr{S}(\mathcal{T})$ is not free.

Suppose that $\mathscr{S}(\mathcal{T})$ is not free, i. e. that there are $\boldsymbol{p}^{\prime}, \boldsymbol{q}^{\prime} \in Q^{+}$with $\boldsymbol{p}^{\prime} \neq \boldsymbol{q}^{\prime}$ but $\boldsymbol{p}^{\prime} \circ=\boldsymbol{q}^{\prime}$ 。 We are done if we have $\left|\boldsymbol{p}^{\prime}\right|=\left|\boldsymbol{q}^{\prime}\right|$. Otherwise, we distinguish two cases: $\boldsymbol{p}^{\prime} \boldsymbol{q}^{\prime} \neq \boldsymbol{q}^{\prime} \boldsymbol{p}^{\prime}$ and $\boldsymbol{p}^{\prime} \boldsymbol{q}^{\prime}=\boldsymbol{q}^{\prime} \boldsymbol{p}^{\prime}$. In the former one, we can set $\boldsymbol{p}=\boldsymbol{p}^{\prime} \boldsymbol{q}^{\prime}$ and $\boldsymbol{q}=\boldsymbol{q}^{\prime} \boldsymbol{p}^{\prime}$. The latter case is a bit more complicated: as $\boldsymbol{p}^{\prime}$ and $\boldsymbol{q}^{\prime}$ commute, there is a word $\boldsymbol{r} \in Q^{+}$such that $\boldsymbol{p}^{\prime}=\boldsymbol{r}^{k}$ and $\boldsymbol{q}^{\prime}=\boldsymbol{r}^{\ell}$ for some $k, \ell \in \mathbb{N}$ (see, for example, [29, p. 8, Porposition 1.3.2]). Without loss of generality, we may assume $k<\ell$. We have id $=\overline{\boldsymbol{p}}^{\prime} \boldsymbol{p}^{\prime} \circ=\overline{\boldsymbol{r}}^{k} \boldsymbol{q}^{\prime} \circ=\overline{\boldsymbol{r}}^{k} \boldsymbol{r}^{\ell} \circ=\boldsymbol{r}^{\ell-k}$ 。, i. e. that $\boldsymbol{r}^{\ell-k} \in Q^{+}$acts like the identity. Since we have $|Q| \geq 2$, there is a state $q \in Q$ which differs from the first letter of $\boldsymbol{r}$ (seen as a word over $Q$ ). Thus, we have $q \boldsymbol{r}^{\ell-k} \neq \boldsymbol{r}^{\ell-k} q$ but $\left|q^{\ell-k}\right|=\left|\boldsymbol{r}^{\ell-k} q\right|$ and $q \boldsymbol{r}^{\ell-k} \circ=\boldsymbol{r}^{\ell-k} q \circ$.

\section{The Finiteness Problem for Invertible, Bi-Reversible Automata}

In this section, we consider Group Finiteness, the finiteness problem for automaton groups:

$\begin{array}{ll}\text { Input: } & \text { a } G \text {-automaton } \mathcal{T} \\ \text { Question: } & \text { is } \mathscr{G}(\mathcal{T}) \text { finite? }\end{array}$

Although it has been studied widely, the decidability of this problem is still an open problem. Notice, however, that the problem is semi-decidable in the sense that there is an algorithm which stops if and only if $\mathscr{G}(\mathcal{T})$ is finite. For this algorithm, one can use the

\footnotetext{
${ }^{15}$ Problems similar to the one presented here are also discussed in 35 .
} 
naïve approach of enumerating all state sequences (in order for ascending length) until no further new group elements are found. The result closest to proving undecidability of Group Finiteness is due to Gillibert, who showed that the finiteness problem for automaton semigroups is undecidable [20, and the recent result on the undecidability of the order problem for automaton groups (checking whether a group element has finite order) [21].

In this section, we extend this result to get closer to automaton groups: we show that the finiteness problem for automaton semigroups remains undecidable if the input automaton is inverse-deterministic and bi-reversible. In particular, we obtain undecidability of the problem whether a subsemigroup of an automaton-inverse semigroup given by some generating states is finite. We obtain these results by connecting the existence of an infinite orbit in the boundary to the finiteness of the semigroup.

Orbital Graphs and Finiteness. We start by characterizing finite semigroups by their dynamics on the boundary. Finiteness of an automaton semigroup is related to the sizes of its orbital graphs as we have the following result that naturally extends the group case [14, Corollary 1].

Proposition 4.1. Let $\mathcal{T}=(Q, \Sigma, \delta)$ be an $S$-automaton. Then, its generated semigroup $\mathscr{S}(\mathcal{T})$ is finite if and only if there exists a constant $C$ such that, for every $\xi \in \Sigma^{\omega}$, the size of $Q^{*} \circ \xi$ is bounded by $C$.

Proof. Suppose that $\mathscr{S}(\mathcal{T})$ is finite. Then, clearly, $\left|Q^{*} \circ \xi\right| \leq|\mathscr{S}(\mathcal{T})|$ for all $\xi \in \Sigma^{\omega}$.

Conversely, since $Q$ is a finite set and there is a constant $C$ such that $\left|Q^{*} \circ \xi\right| \leq C$ for all $\xi \in \Sigma^{\omega}$, there are only finitely many possibilities for the orbital graph $\mathcal{T} \circ \xi$ up to edge-label preserving isomorphism of rooted graphs. For each of these isomorphism classes, we fix a representative. Let them be $\mathcal{T} \circ \xi_{1}, \ldots, \mathcal{T} \circ \xi_{n}$ and let $\mathcal{U}$ be the union of these orbital graphs. Notice that $\mathcal{U}$, as a finite union of finite graphs, is finite itself. Additionally, we have a partial action of $Q^{+}$on $\mathcal{U}$ : For any $\boldsymbol{q} \in Q^{+}$with $\boldsymbol{q}=q_{\ell} \ldots q_{1}$ for $q_{1}, \ldots, q_{\ell} \in Q$, let $\boldsymbol{q} \star u$ denote the vertex of $\mathcal{U}$ reached by following the (unique if existing) path

$$
u \stackrel{q_{1}}{\longrightarrow} q_{1} \circ u \stackrel{q_{2}}{\longrightarrow} q_{2} q_{1} \circ u \stackrel{q_{3}}{\longrightarrow} \cdots \stackrel{q_{\ell}}{\longrightarrow} q_{\ell} \ldots q_{1} \circ u=\boldsymbol{q} \star u
$$

of $\mathcal{U}$ which starts in $u$ and has label $q_{1} \ldots q_{\ell}$. The partial maps $\boldsymbol{q} \star$ mapping a vertex $u$ to $\boldsymbol{q} \star u$ form a semigroup $T$, which is finite since there are only finitely many partial maps from the finite vertex set of $\mathcal{U}$ to itself.

We show that $\mathscr{S}(\mathcal{T})$ is isomorphic to $T$ via the isomorphism $\varphi: \mathscr{S}(\mathcal{T}) \rightarrow T, \boldsymbol{q} \circ \mapsto \boldsymbol{q}$ * First, we have to show that $\varphi$ is well-defined. For this, we need to show that $\boldsymbol{q} \circ=\boldsymbol{p} \circ$ implies $\boldsymbol{q} \star=\boldsymbol{p} \star$. Consider $\boldsymbol{q}=q_{m} \ldots q_{1}$ and $\boldsymbol{p}=p_{\ell} \ldots p_{1}$ for $q_{1}, \ldots, q_{m}, p_{1}, \ldots, p_{\ell} \in Q$ and suppose that there is some $\xi \in \Sigma^{\omega}$ such that $\boldsymbol{q} \star$ differs from $\boldsymbol{p} \star$ on $\xi$. There are two cases: either one of them (say: $\boldsymbol{p} \star$ ) is undefined on $\xi$ or they are both defined but their values differs. In the first case, there is some path in $\mathcal{U}$ which starts in $\xi$ and is labeled by $q_{1} \ldots q_{m}$; on the other hand, there is no such path labeled with $p_{1} \ldots p_{\ell}$. Since $\mathcal{U}$ arises as a union of orbital graphs the (non-)existence of these paths mean that $\boldsymbol{q} \circ$ is 
defined on $\xi$ while $\boldsymbol{p} \circ$ is not. For the second case, we have that both paths exist but end in different nodes. For the same reasons, this means that $\boldsymbol{q} \circ$ and $\boldsymbol{p} \circ$ are both defined on $\xi$ but differ in value.

As surjectivity of $\varphi$ is trivial, it remains to show injectivity. For two distinct elements, $\boldsymbol{q} \circ$ and $\boldsymbol{p} \circ$ of $\mathscr{S}(\mathcal{T})$, there is an $\omega$-word $\xi \in \Sigma^{\omega}$ such that $\boldsymbol{q} \circ$ and $\boldsymbol{p} \circ$ differ on $\eta$ (either because only one of them is defined or because their respective values differ). Let $\mathcal{T} \circ \xi_{i}$ be the orbital graph isomorphic to $\mathcal{T} \circ \xi$. Then, we have $\boldsymbol{q} \star \neq \boldsymbol{p} \star$ because there either is (without loss of generality) only a path belonging to $\boldsymbol{q}$ which starts in $\xi_{i}$ in $\mathcal{U}$ but none belonging to $\boldsymbol{p}$ or both paths exist but they end in different nodes.

Notice that the same idea can be used to prove a similar result for automaton-inverse semigroups:

Proposition 4.2. Let $\mathcal{T}=(Q, \Sigma, \delta)$ be an $\bar{S}$-automaton. Then, its generated inverse semigroup $\overline{\mathscr{S}}(\mathcal{T})$ is finite if and only if there exists a constant $C$ such that, for every $\xi \in \Sigma^{\omega}$, the size of $\tilde{Q}^{*} \circ \xi$ is bounded by $C$, where $\tilde{Q}$ denotes the disjoint union of the states of $\mathcal{T}$ and the states of its inverse $\overline{\mathcal{T}}$.

Notice that it does not suffice that the sizes of all $Q^{*} \circ \xi$ are bounded by some constant for an automaton-inverse semigroup to be finite; we also need to consider the inverses. A counter-example to this has already been given in Lemma 2.6 ${ }^{16}$

Given an infinite automaton semigroup, Proposition 4.1 states that, for every $n \in \mathbb{N}$, there is a word whose orbit is larger than $n$. While it seems plausible that one can obtain a single $\omega$-word with an infinite orbit from this increasing sequence, it is not obvious how this can be done. For example, consider the $S$-automaton

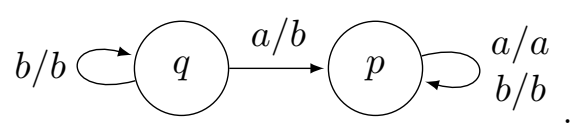

While $p$ acts like the identity, the action of $q$ is to replace the first $a$ in the input word with $b$. Thus, the orbit of $a^{n}$ contains $n+1$ words. However, the same is true for $b^{n} a^{n}$. Now, while the first sequence $\left(a^{n}\right)_{n \in \mathbb{N}}$ converges (with respect to the prefix metric) to $a^{\omega}$, the second sequence $\left(b^{n} a^{n}\right)_{n \in \mathbb{N}}$ converges to $b^{\omega}$. The former indeed has an infinite orbit. The orbit of the latter, however, only contains the word $b^{\omega}$ itself. This example demonstrates that we cannot simply take the limit point of an arbitrary sequence of words with increasing orbit size and obtain a(n $\omega$-)word with an infinite orbit, which leads us to the following question.

Open Problem 4.3. ${ }^{17}$ Let $\mathcal{T}=(Q, \Sigma, \delta)$ be an $S$-automaton. Is $\mathscr{S}(\mathcal{T})$ infinite if and only if there is an $\omega$-word $\xi \in \Sigma^{\omega}$ with an infinite orbit $Q^{*} \circ \xi$ ?

If $\mathcal{T}$ is a $G$-automaton, is $\mathscr{G}(\mathcal{T})$ then infinite if and only if there is an $\omega$-word $\xi \in \Sigma^{\omega}$ with an infinite orbit $\tilde{Q}^{*} \circ \xi$ (where $\tilde{Q}$ is the disjoint union of the states of $\mathcal{T}$ and the states of its inverse)?

\footnotetext{
${ }^{16}$ This stops us from directly transferring our proof for the undecidability of the finiteness problem for invertible, reversible automata to the finiteness problem for automaton-inverse semigroups.

${ }^{17}$ Shortly after making the first version of this paper available on the arXiv (arXiv:1712.07408v1), this problem was solved independently by the authors and Dominik Francoeur [18], which resulted in a joint paper on this topic merging the two proofs [1].
} 
A positive answer to the semigroup case leads to a positive answer for the group case by Lemma 2.5 since $\mathscr{S}(\mathcal{T})$ is finite if and only if so is $\mathscr{G}(\mathcal{T})[1$.

Wang Tilings and Automata. Recall that a Wang tile is a unit square tile with a color on each edge. Formally, it is a quadruple $t=\left(t_{W}, t_{S}, t_{E}, t_{N}\right) \in C^{4}$ where $C$ is a finite set of colors. The choice of $W, S, E$ and $N$ stems from the four cardinal directions and we say, for example, that $t_{W}$ is the color at the west side of $t$. When seeing it as a Wang tile, we write the tuple $t=\left(t_{W}, t_{S}, t_{E}, t_{N}\right)$ as $t=t_{W}^{t_{W}} t_{t_{S}} t_{E}$.

A tile set is a finite set $\mathcal{W}$ of Wang tiles. For each $t \in \mathcal{W}$ and $D \in\{W, S, E, N\}$, we let $t_{D}$ denote the color of the edge on the $D$-side. A tile set $\mathcal{W}$ tiles the discrete plane $\mathbb{Z}^{2}$ if there is a map $f: \mathbb{Z}^{2} \rightarrow \mathcal{W}$ that associates to each point in the discrete plane a tile from $\mathcal{W}$ such that adjacent tiles share the same color on their common edge, i. e. $f(x, y)_{E}=f(x+1, y)_{W}$ and $f(x, y)_{N}=f(x, y+1)_{S}$ for every $(x, y) \in \mathbb{Z}^{2}$. Such a map is called a $\mathbb{Z}^{2}$-tiling. Analogously, a tile set $\mathcal{W}$ tiles the first quadrant of the discrete plane $\mathbb{N}^{2}$ if it admits an $\mathbb{N}^{2}$-tiling, i. e. there is a map $g: \mathbb{N}^{2} \rightarrow \mathcal{W}$ with $g(x, y)_{E}=g(x+1, y)_{W}$ and $g(x, y)_{N}=g(x, y+1)_{S}$ for every $(x, y) \in \mathbb{N}^{2}$.

A $\mathbb{Z}^{2}$-tiling $f$ is called periodic if there exists a (non-zero) periodicity vector $\boldsymbol{v} \in \mathbb{Z}^{2}$ for $f$, i. e. we have $f(\boldsymbol{t}+\boldsymbol{v})=f(\boldsymbol{t})$ for all $t \in \mathbb{Z}^{2}$. If a tile set does not admit a periodic $\mathbb{Z}^{2}$-tiling, it is called aperiodic.

For an $\mathbb{N}^{2}$-tiling $g$, we may define the notion of horizontal words: the $i^{\text {th }}$ horizontal word of $g$ is $f(0, i)_{S} f(1, i)_{S} \ldots$, i. e. the $\omega$-word over $C$ given by the south colors of the rectangle $[0, \infty] \times[i, i]$. We call $g$-recurrent if there are $i \neq j$ such that the $i^{\text {th }}$ and $j^{\text {th }}$ horizontal word of $g$ coincide; if no such $i$ and $j$ exits, we call $g$ non-y-recurrent. The notions of y-recurrence and periodicity of a tile set are linked ${ }^{18}$

Lemma 4.4. A tile set $\mathcal{W}$ admits a y-recurrent $\mathbb{N}^{2}$-tiling if and only if it admits a periodic $\mathbb{Z}^{2}$-tiling.

Proof. Let $g$ be a y-recurrent $\mathbb{N}^{2}$-tiling for $\mathcal{W}$. Then, by definition, there are $i<j$ such that the $i^{\text {th }}$ horizontal word is the same as the $j^{\text {th }}$ one, i. e. the rectangle $[0, \infty] \times[i, j-1]$ is colored the same way on its north and on its south side. Notice that there are only finitely many possible colorings for the west sides of the rectangles $[k, \infty] \times[i, j-1]$ for $k \in \mathbb{N}$. Therefore, there must be $k, \ell \in \mathbb{N}$ with $k<\ell$ such that the colorings on the west side of the respective rectangles coincide, or, in other words, such that the rectangle $[k, \ell-1] \times[i, j-1]$ has the same coloring on its west and its east side. Thus, we can repeat the tile pattern associated to this rectangle infinitely often, both, horizontally and vertically, which yields a periodic $\mathbb{Z}^{2}$-tiling.

For the other direction, let $f$ be a periodic $\mathbb{Z}^{2}$-tiling for $\mathcal{W}$. By an argument similar to the one just presented, one can see that $f$ can be assumed to be vertically periodic, i. e. that there is a $v_{y}>0$ such that $f(x, y)=f\left(x, y+v_{y}\right)$ for all $x, y \in \mathbb{Z}$ (see, for example, [36]). Clearly, the restriction of $f$ into a map $\mathbb{N}^{2} \rightarrow \mathcal{W}$ is a y-recurrent $\mathbb{N}^{2}$-tiling.

Notice that the periodic $\mathbb{Z}^{2}$-tiling might be different from the y-recurrent $\mathbb{N}^{2}$-tiling.

\footnotetext{
${ }^{18}$ The proof is basically the same as the one showing that $\mathcal{W}$ tiles $\mathbb{Z}^{2}$ if and only if it tiles $\mathbb{N}^{2}$ (see [36]).
} 
There is a natural way to associate a (possibly non-deterministic) automaton $\mathcal{T}(\mathcal{W})$ to a tile set $\mathcal{W}$ (and vice-verse, see [12]): the state set and alphabet are the set of colors and we associate a transition $t_{W} \stackrel{t_{S} / t_{N}}{\longrightarrow} t_{E}$ to every tile $\mathcal{W} \ni t=t_{W}^{{ }_{W}} t_{S} t_{E}$. We say that a tile set $\mathcal{W}$ is $C D$-deterministic with $(C, D) \in\{(S, W),(S, E),(N, W),(N, E)\}$ if each tile $t \in \mathcal{W}$ is uniquely determined by its pair $\left(t_{C}, t_{D}\right)$ of colors on the $C$ and $D$ sides. Whenever $\mathcal{W}$ is $C D$-deterministic for each $(C, D) \in\{(S, W),(S, E),(N, W),(N, E)\}$, we say that $\mathcal{W}$ is 4 -way deterministic. There are some obvious connections between the determinism of a tile set and its associated automaton, which we list in the following lemma (see also [12, Lemma 6.1]).

Lemma 4.5. The following facts hold:

- $\mathcal{W}$ is $S W$-deterministic if and only if $\mathcal{T}(\mathcal{W})$ is deterministic;

- $\mathcal{W}$ is $S E$-deterministic if and only if $\mathcal{T}(\mathcal{W})$ is reversible;

- $\mathcal{W}$ is $N W$-deterministic if and only if $\mathcal{T}(\mathcal{W})$ is inverse-deterministic;

- $\mathcal{W}$ is $N E$-deterministic if and only if $\mathcal{T}(\mathcal{W})$ is inverse-reversible.

For aperiodic tile sets, the notion of y-recurrence and the finiteness of the associated semigroup are linked as the following proposition demonstrates.

Proposition 4.6. Let $\mathcal{W}$ be a $S W$-deterministic, aperiodic tile set and let $\mathcal{T}=\mathcal{T}(W)=$ $(Q, \Sigma, \delta)$ be the associated $S$-automaton. Then,

- $\mathscr{S}(\mathcal{T})$ is infinite

- $\mathcal{W}$ admits a $\mathbb{Z}^{2}$-tiling and

- there is an $\omega$-word $\xi \in \Sigma^{\omega}$ with an infinite orbit $Q^{*} \circ \xi$

are equivalent.

Proof. First, we show that a $\mathbb{Z}^{2}$-tiling induces an $\omega$-word with an infinite orbit, which means that $\mathscr{S}(\mathcal{T})$ must be infinite (by Proposition 4.1). Therefore, suppose that $\mathcal{W}$ admits a $\mathbb{Z}^{2}$-tiling $f$. Since $\mathcal{W}$ is aperiodic, its restriction $g$ into an $\mathbb{N}^{2}$-tiling must be non-y-recurrent by Lemma 4.4. Let $\xi_{i}$ denote the $i^{\text {th }}$ horizontal word of $g$ and let $q_{i}=g(0, i)_{W}$ for all $i \in \mathbb{N}$ (i.e. $q_{1} q_{2} \ldots$ labels the west side of the first quadrant read from bottom to top). By construction of $\mathcal{T}$, we have $\xi_{i+1}=q_{i} \circ \xi_{i}$ and, since $g$ is non-y-recurrent, we have $\xi_{i} \neq \xi_{j}$ for all $i \neq j$. Therefore, the orbit $Q^{*} \circ \xi_{0}$ is infinite.

Next, assume that $\mathscr{S}(\mathcal{T})$ is infinite. We show that $\mathcal{W}$ admits a $\mathbb{Z}^{2}$-tiling tiling in this case, which - as shown above - implies that there is an $\omega$-word with an infinite orbit. In fact, we only need to show that $\mathcal{W}$ admits tilings for arbitrarily large squares $[0, \ell] \times[0, \ell]$ since, then, it also admits a $\mathbb{Z}^{2}$-tiling (by a standard compactness argument, see e.g. [36]). By Proposition 4.1 there is an infinite sequence $\left(\xi_{i}\right)_{i \in \mathbb{N}}$ of $\omega$-words such that $\left|Q^{*} \circ \xi_{i}\right| \geq i$, which, by definition, is also the size of the orbital graph $\mathcal{T} \circ \xi_{i}$. Notice 
that, for every $i \in \mathbb{N}$, all nodes in $\mathcal{T} \circ \xi_{i}$ have out-degree at most $|Q|$. Thus, for every $\ell \in \mathbb{N}$, we find an $i(\ell) \in \mathbb{N}$ such that $\mathcal{T} \circ \xi_{i(\ell)}$ contains a path of length $\ell$

$$
\eta^{(\ell)}=\eta_{0}^{(\ell)} \stackrel{q_{1}^{(\ell)}}{\longrightarrow} \eta_{1}^{(\ell)} \stackrel{q_{2}^{(\ell)}}{\longrightarrow} \ldots \stackrel{q_{\ell}^{(\ell)}}{\longrightarrow} \eta_{\ell}^{(\ell)}
$$

where all $\eta_{j}^{(\ell)} \in \Sigma^{\omega}$ are in the orbit $Q^{*} \circ \xi_{i(\ell)}$. Let $u_{j}^{(\ell)}$ denote the prefix of $\eta_{j}^{(\ell)}$ of length $\ell$. Notice that, due to prefix-compatibility, we still have $u_{j}^{(\ell)}=q_{j} \circ u_{j-1}^{(\ell)}$. By construction of $\mathcal{T}=\mathcal{T}(\mathcal{W})$, this yields a tiling of $[0, \ell] \times[0, \ell]$ where the south sides of the $i^{\text {th }}$ row (with $i \in \mathbb{N}$ ) is labeled by $u_{i}^{(\ell)}$, the north side of the $\ell^{\text {th }}$ row is labeled by $u_{\ell}^{(\ell)}$ and the west side of the square is labeled by $q_{1} \ldots q_{\ell}$.

The previous proposition can be used for the reduction to show the main result of this section:

Theorem 4.7. The strengthened version

Input: $\quad$ a bi-reversible and bi-deterministic automaton $\mathcal{T}$

Question: is $\mathscr{S}(\mathcal{T})$ finite?

of the finiteness problem for automaton semigroups remains undecidable. In particular, it is undecidable to check whether an automaton subsemigroup of an automaton-inverse semigroup is finite.

Furthermore, the problem

Input: $\quad$ a bi-reversible and bi-deterministic automaton $\mathcal{T}=(Q, \Sigma, \delta)$

Question: is there an $\omega$-word $\xi \in \Sigma^{\omega}$ with an infinite orbit $Q^{*} \circ \xi$ ?

is undecidable.

Proof. Lukkarila [30] has shown that the tiling problem for Wang tilings remains undecidable when the input is restricted to be a 4 -way deterministic, aperiodic tile sets. In other words, he showed undecidability of the problem:

Input: a 4-way deterministic, aperiodic tile set $\mathcal{W}$

Question: does $\mathcal{W}$ admit a $\mathbb{Z}^{2}$-tiling?

To prove this undecidability, he constructed a 4 -way deterministic tile set from a Turing Machine in such a way that the tile set admits a tiling if and only if the Turing Machine halts. The constructed tiles consist of multiple layers of tiles (using direct products) and the first layer uses the 4-way deterministic, aperiodic tile set of Kari and Papasoglu [26].

For a 4-way-deterministic and aperiodic tile set $\mathcal{W}$, the automaton $\mathcal{T}(\mathcal{W})$ is bi-reversible and bi-deterministic by Lemma 4.5 and, by Proposition 4.6, it generates an infinite semigroup if and only if $\mathcal{W}$ admits a $\mathbb{Z}^{2}$-tiling, which is the case if and only if there is an $\omega$-word with an infinite orbit. Thus, mapping $\mathcal{W}$ to $\mathcal{T}(\mathcal{W})$ is a co-reduction from Lukkarila's problem to our strengthened version of the finiteness problem and a reduction to our second problem.

Unfortunately, the above theorem neither shows undecidability for the finiteness problem of automaton groups nor for the finiteness problem of automaton-inverse semigroups. 
Although, for the former, we have that $\mathscr{G}(\mathcal{T})$ is infinite if and only if $\mathscr{S}(\mathcal{T})$ is infinite, the automaton associated to a 4 -way deterministic tile set is not necessarily complete and, thus, not a $G$-automaton. This incompleteness is inherent to the undecidability of the tiling problem: the automaton is complete if and only if, for every pair $\left(c_{S}, c_{W}\right)$ of colors, there is at least (and, thus, due to 4-way determinism exactly) one tile whose south side is colored with $c_{S}$ and whose west side is colored with $c_{W}$. If this is true for some tile set, however, it always admits a $\mathbb{N}^{2}$-tiling as one can choose the colors for the $\mathrm{x}$-axis and for the $\mathrm{y}$-axis arbitrarily and continue the tiling from there.

One can complete an $S$-automaton by adding a sink state (see [16, Proposition 1]). This has the effect of (possibly ${ }^{19}$ ) adding a zero to the generated semigroup and, therefore, maintains (in)finiteness. However, it does not maintain reversibility, which leads to the following open problem.

Open Problem 4.8. Given a reversible $S$-automaton, can one compute a complete and reversible $S$-automaton $\mathcal{T}^{\prime}$ such that $\mathscr{S}(\mathcal{T})$ is finite if and only if $\mathscr{S}\left(\mathcal{T}^{\prime}\right)$ is finite? Is it possible to give such a construction if $\mathcal{T}$ is a bi-reversible $\bar{S}$-automaton?

A positive answer to this open problem would lead to the undecidability of the finiteness problem for automaton groups. Indeed, for the reduction, one could take the bi-reversible and bi-deterministic automaton $\mathcal{T}$ obtained from the 4 -way-deterministic tile set in Theorem 4.7, compute its reversible completion $\mathcal{T}^{\prime}$ and take the dual of $\mathcal{T}^{\prime}$, which is a $G$-automaton. Then, we had

$$
|\mathscr{S}(\mathcal{T})|=\infty \Longleftrightarrow\left|\mathscr{S}\left(\mathcal{T}^{\prime}\right)\right|=\infty \Longleftrightarrow\left|\mathscr{S}\left(\partial \mathcal{T}^{\prime}\right)\right|=\infty \Longleftrightarrow\left|\mathscr{G}\left(\partial \mathcal{T}^{\prime}\right)\right|=\infty
$$

where the first equivalence is due to the assumption in the open problem. For the second equivalence, see e.g. [14, Corollary 1] or [1] and, for the third equivalence see [1].

For automaton-inverse semigroups, on the other hand, the partiality is not problematic. Here, the problem is rather that we have to consider the inverses as well. From the perspective of Wang tiles, taking the inverse belongs to mirroring a tile at the horizontal axis. The union of the tiles and their mirrored versions is not an aperiodic tile set anymore. In fact, it will always admit an $\mathbb{N}^{2}$-tiling as long as we can tile a single rightinfinite row. In this case, our approach of obtaining an infinite orbit cannot be applied, which leaves us with the following open problem.

Open Problem 4.9. Is the finiteness problem for automaton-inverse semigroups

Input: $\quad$ an $\bar{S}$-automaton

Question: is $\overline{\mathscr{S}}(\mathcal{T})$ finite?

decidable?

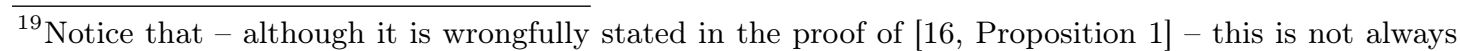
the case; see [17, Section 3] for a discussion.
} 


\section{References}

[1] Ali Akhavi, Ines Klimann, Sylvain Lombardy, Jean Mairesse, and Matthieu Picantin. On the finiteness problem for automaton (semi)groups. International Journal of Algebra and Computation, 22(06):1-26, 2012.

[2] Laurent Bartholdi and Ivan Mitrofanov. The word and order problems for self-similar and automata groups. arXiv preprint arXiv:1710.10109, 2017.

[3] James Belk and Collin Bleak. Some undecidability results for asynchronous transducers and the Brin-Thompson group $2 \mathrm{~V}$. Transactions of the American Mathematical Society, 369(5):3157-3172, 2017.

[4] Paul C. Bell and Igor Potapov. On the undecidability of the identity correspondence problem and its applications for word and matrix semigroups. International Journal of Foundations of Computer Science, 21(06):963-978, 2010.

[5] Ievgen V. Bondarenko, Natalia Bondarenko, Said Sidki, and Flavia Zapata. On the conjugacy problem for finite-state automorphisms of regular rooted trees. Groups, Geometry, and Dynamics, 7(2):323-355, 2013.

[6] Tara Brough and Alan J. Cain. Automaton semigroup constructions. Semigroup Forum, 90(3):763-774, 2015.

[7] Tara Brough and Alan J. Cain. Automaton semigroups: new constructions results and examples of non-automaton semigroups. Theoretical Computer Science, 674:1$15,2017$.

[8] Andrew M. Brunner and Said Sidki. The generation of $G L(n, \mathbb{Z})$ by finite state automata. International Journal of Algebra and Computation, 08(01):127-139, 1998.

[9] Alan J. Cain. Automaton semigroups. Theoretical Computer Science, 410(47):50225038, 2009.

[10] Daniele D'Angeli, Alfredo Donno, Michel Matter, and Tatiana Nagnibeda. Schreier graphs of the Basilica group. J. Mod. Dyn., 4(1):167-205, 2010.

[11] Daniele D'Angeli, Dominik Francoeur, Emanuele Rodaro, and Jan Philipp Wächter. Orbits of automaton semigroups and groups. arXiv preprint arXiv:1903.00222, 2019.

[12] Daniele D'Angeli, Thibault Godin, Ines Klimann, Matthieu Picantin, and Emanuele Rodaro. Boundary action of automaton groups without singular points and Wang tilings. arXiv preprint arXiv:1604.07736, 2016.

[13] Daniele D'Angeli and Emanuele Rodaro. Groups and semigroups defined by colorings of synchronizing automata. International Journal of Algebra and Computation, 24(06):773-793, 2014. 
[14] Daniele D'Angeli and Emanuele Rodaro. A geometric approach to (semi)-groups defined by automata via dual transducers. Geometriae Dedicata, 174(1):375-400, 2015.

[15] Daniele D'Angeli and Emanuele Rodaro. Freeness of automaton groups vs boundary dynamics. Journal of Algebra, 462:115-136, 2016.

[16] Daniele D'Angeli, Emanuele Rodaro, and Jan Philipp Wächter. On the complexity of the word problem for automaton semigroups and automaton groups. Advances in Applied Mathematics, 90:160 - 187, 2017.

[17] Daniele D'Angeli, Emanuele Rodaro, and Jan Philipp Wächter. On the structure theory of partial automaton semigroups. arXiv preprint arXiv:1811.09420, 2018.

[18] Dominik Francoeur. Infinite finitely generated automata semigroups have infinite orbits. arXiv preprint arXiv:1801.10009, 2018.

[19] Dominik Francoeur and Ivan Mitrofanov. On the existence of free subsemigroups in reversible automata semigroups. arXiv preprint arXiv:1811.04679, 2018.

[20] Pierre Gillibert. The finiteness problem for automaton semigroups is undecidable. International Journal of Algebra and Computation, 24(01):1-9, 2014.

[21] Pierre Gillibert. An automaton group with undecidable order and engel problems. Journal of Algebra, 497:363 - 392, 2018.

[22] Thibault Godin, Ines Klimann, and Matthieu Picantin. On torsion-free semigroups generated by invertible reversible Mealy automata. In Adrian-Horia Dediu, Enrico Formenti, Carlos Martín-Vide, and Bianca Truthe, editors, Language and Automata Theory and Applications (LATA 2015), volume 8977 of LNCS, pages 328339. Springer, Berlin, 2015. Arxiv:1410.4488.

[23] Rostislav I. Grigorchuk, Volodymyr V. Nekrashevych, and Vitaly I. Sushchansky. Automata, dynamical systems, and groups. Proc. Steklov Inst. Math., 231(4):128203, 2000 .

[24] Mikhail Gromov. Metric Structures for Riemannian and Non-Riemannian Spaces. Modern Birkhäuser Classics. Birkhäuser Basel, 1 edition, 2007. Originally published as volume 152 in the series: Progress in Mathematics, Original French edition published with the title: Structures Métriques des Variétés Riemanniennes.

[25] John M. Howie. Fundamentals of Semigroup Theory. London Mathematical Society Monographs. Clarendon Press, 1996.

[26] Jarkko Kari and Panagiotis Papasoglu. Deterministic aperiodic tile sets. Geometric Ef Functional Analysis, 9(2):353-369, 1999. 
[27] David A. Klarner, Jean-Camille Birget, and Wade Satterfield. On the undecidability of the freeness of integer matrix semigroups. International Journal of Algebra and Computation, 1(2):223-226, 1991.

[28] Ines Klimann. The finiteness of a group generated by a 2-letter invertible-reversible mealy automaton is decidable. In N. Portier and T. Wilke, editors, 30th Int. Symp. on Theoretical Aspects of Computer Science, Kiel, Germany, volume 20 of Leibniz International Proceedings, pages 502-513, 2013.

[29] M. Lothaire. Combinatorics on Words, volume 17 of Encyclopedia of Mathematics and Its Applications. Addison-Wesley, 1983.

[30] Ville Lukkarila. The 4-way deterministic tiling problem is undecidable. Theoretical Computer Science, 410(16):1516 - 1533, 2009.

[31] Volodymyr V. Nekrashevych. Self-similar inverse semigroups and Smale spaces. International Journal of Algebra and Computation, 16(5):849-874, 2006.

[32] Andrij S. Olijnyk, Vitaly I. Sushchansky, and Janusz K. Słupik. Inverse semigroups of partial automaton permutations. International Journal of Algebra and Computation, 20(07):923-952, 2010.

[33] Feyishayo Olukoya. The growth rates of automaton groups generated by reset automata. arXiv preprint arXiv:1708.07209, 2017.

[34] Mario Petrich. Inverse Semigroups. Pure \& Applied Mathematics. John Wiley \& Sons Inc, 1984.

[35] Alan Pierce. Decision problems on iterated length-preserving transducers. Senior Thesis, 2011.

[36] Raphael M. Robinson. Undecidability and nonperiodicity for tilings of the plane. Inventiones mathematicae, 12(3):177-209, 1971.

[37] Pedro V. Silva and Benjamin Steinberg. On a class of automata groups generalizing lamplighter groups. International Journal of Algebra and Computation, 15(05n06):1213-1234, 2005.

[38] Zoran Šunić and Enric Ventura. The conjugacy problem in automaton groups is not solvable. Journal of Algebra, 364:148-154, 2012.

[39] Yaroslav Vorobets. Notes on the Schreier graphs of the Grigorchuk group. In Dynamical systems and group actions, volume 567 of Contemp. Math., pages 221-248. Amer. Math. Soc., Providence, RI, 2012.

[40] Andrzej Zuk. Automata groups. Topics in Noncommutative Geometry, 16:165, 2012. 


\title{
Erratum to "Automaton Semigroups and Groups: On the Undecidability of Problems Related to Freeness and Finiteness"
}

\author{
Daniele D’Angeli*1 ${ }^{*}$ Emanuele Rodaro ${ }^{\dagger 2}$ and Jan Philipp Wächter ${ }^{3}$ \\ ${ }^{1}$ Università degli Studi Niccolò Cusano, Via Don Carlo Gnocchi, 3, 00166 Roma, Italy \\ 2 Department of Mathematics, Politecnico di Milano, Piazza Leonardo da Vinci, 32, 20133 Milano, Italy \\ ${ }^{3}$ Institut für Formale Methoden der Informatik (FMI), Universität Stuttgart, Universitätsstraße 38, 70569 Stuttgart, Germany
}

\section{April 9, 2020}

The last part of Lemma 3.5 about the generated group is stated wrongly. Independently from that, the argumentation below Corollary 3.8 is flawed. This affects Proposition 3.9, Theorem 3.12, Corollary 3.13 and Corollary 3.14. In particular, the question whether the freeness problem for automaton semigroups is decidable remains open.

Lemma 3.5. The last part of Lemma 3.5 states that the group generated by the automaton $\mathcal{T}_{\mathcal{M}}$ is isomorphic to a subgroup of $\operatorname{Aff}_{d}(\mathbb{Z})$. However, in general, the inverse over $\mathbb{Z}_{n}$ of a matrix with entries from $\mathbb{Z}$ is not from $\mathbb{Z}^{d \times d}$. In fact, this only holds for matrices with determinant -1 or 1 . Thus, the group generated by $\mathcal{T}_{\mathcal{M}}$ is only isomorphic to a subgroup of $\operatorname{Aff}_{d}\left(\mathbb{Z}_{n}\right)$.

The Group Case. Generally, the problem is that not every relation in $\mathcal{T}_{\mathcal{M}}$ yields a relation in the linear (semi)group generated by some finite set of matrices $\mathcal{M}$ (where $\mathcal{T}_{\mathcal{M}}$ is the automaton from Lemma 3.5). A relation of the form

$$
m_{M_{\ell}, \boldsymbol{v}_{\ell}} \ldots m_{M_{1}, \boldsymbol{v}_{1}} \circ=m_{M_{\ell}, \boldsymbol{v}_{\ell}^{\prime}} \ldots m_{M_{1}, \boldsymbol{v}_{1}^{\prime}} \circ
$$

with $\ell \geq 1, M_{1}, \ldots M_{\ell} \in \mathcal{M}$ and $\boldsymbol{v}_{i}, \boldsymbol{v}_{i}^{\prime} \in V_{M_{i}}$ for all $1 \leq i \leq \ell$ can exist for $\mathcal{T}_{\mathcal{M}}$. It is a proper relation if there is some $1 \leq i \leq \ell$ with $\boldsymbol{v}_{i} \neq \boldsymbol{v}_{i}^{\prime}$. Such a proper relation implies

$$
M_{\ell} \ldots M_{1}=M_{\ell} \ldots M_{1}
$$

in the linear semigroup by Lemma 3.6; however, this is not a proper relation.

\footnotetext{
${ }^{*}$ The first author was supported by the Austrian Science Fund project FWF P24028-N18 and FWF P29355-N35.

${ }^{\dagger}$ The second author thanks the project INDAM-GNSAGA.
} 
Concerning Proposition 3.9, we can prove that, in fact, the group generated by $\mathcal{T}_{\mathcal{M}}$ is never free (except for trivial cases):

Proposition A. Let $d \geq 2$ and $\mathcal{M}$ be a finite set of $\mathbb{Z}^{d \times d}$ matrices with non-zero determinant. Then, $\mathscr{G}\left(\mathcal{T}_{\mathcal{M}}\right)$ is not free (where $\mathcal{T}_{\mathcal{M}}$ is the $G$-automaton from Lemma 3.5).

Proof. The group generated by $\mathcal{T}_{\mathcal{M}}$ is isomorphic to the subgroup $G$ of $\operatorname{Aff}_{d}\left(\mathbb{Z}_{n}\right)$ generated by $\left\{M_{\boldsymbol{v}} \mid M \in \mathcal{M}, \boldsymbol{v} \in V_{M}\right\}$. We will show that this affine subgroup is not free. Instead of $M_{\boldsymbol{v}}$, we will use the semidirect product notation $(\boldsymbol{v}, M)$ for elements of $G$.

First, observe that the linear group $L$ generated by $\mathcal{M}$ is a subgroup of $G$. If it is trivial, then $\mathcal{M}$ can only contain the identity matrix and, by [4, Lemma 4.1], we have $G \simeq \mathbb{Z}^{d} \rtimes L$. Since we have $d \geq 2$, this contains the non-cyclic, abelian subgroup $\mathbb{Z}^{d}$ and, thus, cannot be free.

Therefore, assume that $L$ is non-trivial and let $1 \neq M \in L$. The group $G$ cannot be free if $L$ is not free and there is nothing to show in this case. Thus, we assume that $L$ is free, which implies that all (non-identity) matrices in $\mathcal{M}$ have infinite order (as elements of the general linear group). Let $\boldsymbol{u}$ and $\boldsymbol{v}$ be two different vectors from $V_{M}$ (note that such vectors always exist) and consider the subgroup $H$ of $G$ generated by $(\boldsymbol{u}, M)$ and $(\boldsymbol{v}, M)$. We will show that this subgroup is solvable but not cyclic and, therefore, not free.

First, assume that $H$ is cylic. Then, there is some $(\boldsymbol{w}, N)$ such that $(\boldsymbol{u}, M)=(\boldsymbol{w}, N)^{i}$ and $(\boldsymbol{v}, M)=(\boldsymbol{w}, N)^{j}$ for some $i, j \in \mathbb{Z}$. From the second components, we obtain $N^{i}=M=N^{j}$. Since $M$ is not the identity matrix, $N$ cannot be the identity matrix either and must, therefore, have infinite order. This implies $i=j$, which is not possible, however, as we have $\boldsymbol{u} \neq \boldsymbol{v}$.

To show that $H$ is solvable, we show that the commutator subgroup $[H, H]$ is abelian. To see this, consider a commutator $\left[\left(\boldsymbol{w}_{1}, M\right),\left(\boldsymbol{w}_{2}, M\right)\right]$ of elements $\left(\boldsymbol{w}_{1}, M\right),\left(\boldsymbol{w}_{2}, M\right) \in$ $H$. We have

$$
\left[\left(\boldsymbol{w}_{1}, M\right),\left(\boldsymbol{w}_{2}, M\right)\right]=\left(\boldsymbol{w}_{2}, M\right)^{-1}\left(\boldsymbol{w}_{1}, M\right)^{-1}\left(\boldsymbol{w}_{2}, M\right)\left(\boldsymbol{w}_{1}, M\right)=\left(\boldsymbol{w}^{\prime}, 1\right)
$$

for some vector $\boldsymbol{w}^{\prime}$. Thus, all such transformations commute.

As a consequence, Proposition 3.9 can only be stated as follows.

Proposition 3.9. IICP is reducible to the problem

Input: $\quad a$ G-automaton $\mathcal{T}=(Q, \Sigma, \delta)$ and

$$
\text { a finite subset } \boldsymbol{P} \subseteq Q^{+}
$$

Question: does the subgroup generated by $\boldsymbol{P}$ in $\mathscr{G}(\mathcal{T})$ admit a relation over $\boldsymbol{P}$ ?

However, the order problem for automaton groups

Input: $\quad$ a $G$-automaton $\mathcal{T}=(Q, \Sigma, \delta)$ and

some $\boldsymbol{q} \in Q^{*}$

Question: has $\boldsymbol{q}$ finite order in $\mathscr{G}(\mathcal{T})$ ?

is a special case of the above problem. Therefore, the undecidability of the latter follows (independently from the undecidability of IICP) from the undecidability of the former shown by Gillibert [2]. 
The Semigroup Case. The same problem also affects the semigroup case in Theorem 3.12 and Corollary 3.13. Accordingly, the statement that "by Lemma 3.6, the semigroup generated by $\mathcal{T}_{\mathcal{M}}$ is free if and only if so is the linear semigroup generated by $\mathcal{M}$ " made in the original proof of Theorem 3.12 is incorrect. In fact, we obtain - similar to the group case - that the semigroup generated by $\mathcal{T}_{\mathcal{M}}$ is never free.

Proposition B. Let $\mathcal{M}$ be a non-empty, finite set of matrices from $\mathbb{Z}^{d \times d}$. Then, $\mathscr{S}\left(\mathcal{T}_{\mathcal{M}}\right)$ is not free semigroup (where $\mathcal{T}_{\mathcal{M}}$ is the $S$-automaton from Lemma 3.5).

To prove Proposition B, we show something more general and derive Proposition B from that. However, we first need to introduce some semigroup concepts. To every semigroup $S$, we can adjoin a new neutral element 1 to obtain the monoid $S^{1}$. Clearly, $S$ is a free semigroup if and only if $S^{1}$ is a free monoid.

A proper length function for some monoid $M$ is a homomorphism $\lambda$ from $M$ to the free monoid of rank one $(\mathbb{N},+)$ such that $\lambda(s)=0$ implies $s=1$. A monoid $M$ is equidivisible if, for all $s_{1}, s_{2}, s_{1}^{\prime}, s_{2}^{\prime} \in M$ with $s_{1} s_{2}=s_{1}^{\prime} s_{2}^{\prime}$, there is some $x \in M$ with $s_{1}=s_{1}^{\prime} x$ and $x s_{2}=s_{2}^{\prime}$ or with $s_{1} x=s_{1}^{\prime}$ and $s_{2}=x s_{2}^{\prime}$. Free monoids are equidivisable by Levi's Lemma [3, Proposition 7.1.2] and, obviously, admit a proper length function; the converse holds as well [3, Proposition 7.1.8], which yields the following characterization.

Fact C. A monoid $M$ is free if and only if it admits a proper length function and is equidivisable.

If $T$ is a subsemigroup of a free semigroup $S$, then $T^{1}$ is a submonoid of $S^{1}$ and inherits the proper length function of $S^{1}$. Accordingly, $T$ is free if (and only if) $T^{1}$ is equidivisable.

A (left) action of a semigroup $S$ on a monoid $M$ is a homomorphism $\alpha: S \rightarrow \operatorname{End}(M)$ where $\operatorname{End}(M)$ is the monoid of monoid endomorphisms of $M$. In particular, we require $\alpha_{s}\left(1_{M}\right)=1_{M}$ for all $\alpha_{s}=\alpha(s)$ (with $s \in S$ ). We will also write ${ }^{s} m$ for $\alpha_{s}(m)$ if the action is clear from the context.

If the semigroup $S$ acts on the left on some monoid $M$, we can define the semidirect product $M \rtimes S$ : it is the semigroup whose elements are in $M \times S$ and whose multiplication is given by $(m, s)(n, t)=\left(m^{s} n, s t\right)$. We can consider $S$ as a subsemigroup of $M \rtimes S$ by identifying $s$ with $(1, s){ }^{1}$

Lemma D. Let $M$ be a monoid, $S$ a semigroup and let $T$ be a subsemigroup of $M \rtimes S$ containing $S$ as a subsemigroup. Then, $S$ is a free semigroup if $T$ is one.

Proof. Assume that $T$ is a free semigroup or, equivalently, that $T^{1}$ is a free monoid. Then, $T^{1}$ is equidivisable and has a proper length function. We will show that both these properties are inherited by $S^{1}$ and that, thus, $S$ is a free semigroup.

For the proper length function, we set the length of 1 to 0 and the length of $s$ to that of $(1, s)$, which is in $T$ because $S$ is a subsemigroup of $T$. To show that $S^{1}$ is equidivisable, let $s_{1} s_{2}=s_{1}^{\prime} s_{2}^{\prime}$ for $s_{1}, s_{2}, s_{1}^{\prime}, s_{2}^{\prime} \in S^{1}$. We need some $x \in S^{1}$ with $s_{1}=s_{1}^{\prime} x$ and $x s_{2}=s_{2}^{\prime}$ or with $s_{1} x=s_{1}^{\prime}$ and $s_{2}=x s_{2}^{\prime}$. If we have $s_{1}=1$, we can set $x=s_{1}^{\prime}$ to obtain $s_{1} x=s_{1}^{\prime}$ and $s_{2}=1 s_{2}=s_{1}^{\prime} s_{2}^{\prime}=x s_{2}^{\prime}$. The cases $s_{2}=1, s_{1}^{\prime}=1$ or $s_{2}^{\prime}=1$ are symmetric. In

\footnotetext{
${ }^{1}$ This is possible because we have ${ }^{s} 1=1$.
} 
the case $s_{1}, s_{2}, s_{1}^{\prime}, s_{2}^{\prime} \neq 1$, we have $\left(1, s_{1}\right)\left(1, s_{2}\right)=\left(1, s_{1}^{\prime}\right)\left(1, s_{2}^{\prime}\right)$ in $T^{1}$ and, because $T^{1}$ is equidivisable, there is some $y \in T^{1}$ with $\left(1, s_{1}\right)=\left(1, s_{1}^{\prime}\right) y$ and $y\left(1, s_{2}\right)=\left(1, s_{2}^{\prime}\right)$ or with $\left(1, s_{1}\right) y=\left(1, s_{1}^{\prime}\right)$ and $\left(1, s_{2}\right)=y\left(1, s_{2}^{\prime}\right)$. If $y=1$, we obtain $s_{1}=s_{1}^{\prime}$ and $s_{2}=s_{2}^{\prime}$ and we choose $x=1$. Otherwise, $y \in T^{1} \backslash\{1\}=T \subseteq M \rtimes S$ is of the form $y=(m, x) \in M \times S$ and, from the second components of the equations in the two cases, we see that $x \in S$ is indeed the sought element, which concludes the proof that $S^{1}$ is equidivisable.

Proposition E. Let $M$ be a monoid and $S$ a semigroup. Furthermore, let $T$ be a subsemigroup of $M \rtimes S$ such that $S$ is a proper subsemigroup of $T$. Let $(\hat{m}, \hat{s}) \in T \backslash S$ (i. e. we have $M \ni \hat{m} \neq 1_{M}$ and $\hat{s} \in S$ ).

If $(\hat{s} \hat{m}, \hat{s})$ is in $T$, then $T$ cannot be a free semigroup.

Proof. We show the statement by contradiction. Thus, assume that $T$ is a free semigroup. Then, $S$ is a also a free semigroup by Lemma $\mathrm{D}$ and $T^{1}$ and $S^{1}$ are both free monoids.

Since $S$ is a subsemigroup of $T$, we have $(1, \hat{s}) \in T$ and

$$
(1, \hat{s})(\hat{m}, \hat{s})=\left(1^{\hat{s}} \hat{m}, \hat{s}^{2}\right)=\left({ }^{\hat{s}} \hat{m} 1, \hat{s}^{2}\right)=\left({ }^{\hat{s}} \hat{m}, \hat{s}\right)(1, \hat{s})
$$

because the action of $S$ on the monoid $M$ fixes the neutral element. By assumption, we have $\left({ }^{\hat{s}} \hat{m}, \hat{s}\right) \in T$ and, because $T^{1}$ as a free monoid is equidivisable, there is some $y \in T^{1}$ with $y(\hat{m}, \hat{s})=(1, \hat{s})$ or with $(\hat{m}, \hat{s})=y(1, \hat{s})$. Since we have $\hat{m} \neq 1$, we obtain $y \neq 1$ in both cases. Thus, $y=(m, x) \in T^{1} \backslash\{1\}=T \subseteq M \times S$ and we obtain $(m, x)(\hat{m}, \hat{s})=(1, \hat{s})$ in the first and $(\hat{m}, \hat{s})=(m, x)(1, \hat{s})$ in the second case. In both cases, we have $x \hat{s}=\hat{s}$ in $S \leq S^{1}$. Since $S^{1}$ as a free monoid is cancellative, we obtain $x=1 \notin S$; a contradiction.

Now, we can finally derive Proposition B from Proposition E.

Proof of Proposition B. By Lemma 3.5, the semigroup generated by $\mathcal{T}_{\mathcal{M}}$ is isomorphic to the subsemigroup $T$ of $\mathrm{SAff}_{d}(\mathbb{Z})$ generated by $\left\{M_{\boldsymbol{v}} \mid M \in \mathcal{M}, \boldsymbol{v} \in V_{M}\right\}$. In other words, $T$ is a subsemigroup of the semidirect product $\mathbb{Z}^{d} \rtimes S$ where $S$ is the linear semigroup generated by $\mathcal{M}$ and the action of $S$ on the (additive) monoid $\mathbb{Z}^{d}$ is given by the normal matrix multiplication.

If $\mathcal{M}$ only contains the zero matrix $0 \in \mathbb{Z}^{d \times d}$, then $T$ consists only of $0_{0}$, i. e. $T$ is the trivial monoid and, thus, not a free semigroup. If $\mathcal{M}$ contains a non-zero matrix $M$, then we must have $\|M\| \geq 1$. We will show that there is some $\boldsymbol{v} \in V_{M} \backslash\{\mathbf{0}\}$ with $M_{M \boldsymbol{v}} \in V_{M}$. This will allow us to apply Proposition $\mathrm{E}$ and we obtain that $T$ is not free.

As the vector $\boldsymbol{v}$, we choose $-\boldsymbol{e}_{1}$ where $\boldsymbol{e}_{1}$ is the $1^{\text {st }} d$-dimensional unit vector. We have $\|M\| \geq 1$ and, thus, $-\|M\| \leq-1$, which implies $\boldsymbol{v}=-\boldsymbol{e}_{1} \in V_{M}$. On the other hand, we have that the $i^{\text {th }}$ component of $\boldsymbol{w}=M \boldsymbol{v}$ is $-m_{i, 1}$ where $m_{i, 1}$ is the entry of $M$ in the $i^{\text {th }}$ row and $1^{\text {st }}$ column. Since we have $m_{i, 1} \leq\|M\|$ and, thus, $-m_{i, 1} \geq-\|M\|$, we obtain $\boldsymbol{w}=M \boldsymbol{v} \in V_{M}$ as well.

As a result of the flaw in the argumentation, Theorem 3.12 and Corollary 3.13 have to be re-formulated as follows; in particular, the question whether the freeness problem for automaton semigroups is decidable remains open. 
Theorem 3.12. The problem

Input: $\quad a$ G-automaton $\mathcal{T}=(Q, \Sigma, \delta)$ and

a finite subset $\boldsymbol{P} \subseteq Q^{+}$

Question: is the subsemigroup generated by $\boldsymbol{P}$ in $\mathscr{S}(\mathcal{T})$ free?

is undecidable.

Corollary 3.13. The freeness problem for subsemigroups of automaton semigroups

Input: $\quad$ a finitely generated subsemigroup $T$ of an automaton semigroup

Question: is T free?

is undecidable.

The corrected version of Theorem 3.12 can be proved by reducing MATRIX SEMIGROUP FREENESS to the problem. Here, we map $\mathcal{M}$ to $\mathcal{T}_{\mathcal{M}}$ and use

$$
\boldsymbol{P}=\left\{m_{M, 0} \mid M \in \mathcal{M}\right\}
$$

as the generators of the subsemigroup (where $\mathbf{0}$ is the $d$-dimensional zero vector). The corrected version of Corollary 3.13 immediately follows from the corrected version of Theorem 3.12.

Again, the corrected version of Theorem 3.12 also follows from the undecidability of the order problem for automaton groups [2] (and the corrected version of Corollary 3.13 even follows from the undecidability of the order/torsion problem for automaton semigroups implied by [1, Lemma 3.11 and 3.12]).

Finally, the reduction in the proof of Corollary 3.14 is still correct. However, we do not have that $G$-SEMIGRoup FrEEnEss is undecidable anymore. Therefore, the statement of the corollary does not follow.

\section{References}

[1] Pierre Gillibert. The finiteness problem for automaton semigroups is undecidable. International Journal of Algebra and Computation, 24(01):1-9, 2014.

[2] Pierre Gillibert. An automaton group with undecidable order and engel problems. Journal of Algebra, 497:363 - 392, 2018.

[3] John M. Howie. Fundamentals of Semigroup Theory. London Mathematical Society Monographs. Clarendon Press, 1996.

[4] Zoran Šunić and Enric Ventura. The conjugacy problem in automaton groups is not solvable. Journal of Algebra, 364:148-154, 2012. 